\title{
Transcriptome Analysis Reveals the Symbiotic Mechanism of Ustilago esculenta-Induced Gall Formation of Zizania latifolia
}

\author{
Jie Li, ${ }^{1,2}$ Zhiyuan Lu, ${ }^{1,2}$ Yang Yang, ${ }^{1,2}$ Jinfeng Hou, ${ }^{1,2,3}$ Lingyun Yuan, ${ }^{1,2,3}$ Guohu Chen, ${ }^{1,2,3}$ \\ Chenggang Wang, ${ }^{1,2,3}$ Shaoke Jia, ${ }^{1,2}$ Xuming Feng, ${ }^{1,2}$ and Shidong Zhu ${ }^{1,2,3, \dagger}$ \\ ${ }^{1}$ Vegetable Genetics and Breeding Laboratory, College of Horticulture, Anhui Agricultural University; Hefei 230036, China \\ ${ }^{2}$ Anhui Provincial Engineering Laboratory of Horticultural Crop Breeding, Hefei 230036, China \\ ${ }^{3}$ Wanjiang Vegetable Industrial Technology Institute, Maanshan 238200, China
}

Accepted 27 October 2020.

\begin{abstract}
Zizania latifolia is a perennial aquatic vegetable, whose symbiosis with the fungus Ustilago esculenta (member of Basidiomycota, class Ustilaginaceae) results in the establishment of swollen gall formations. Here, we analyzed symbiotic relations of $Z$. latifolia and $U$. esculenta, using a triadimefon (TDF) treatment and transcriptome sequencing (RNA-seq). Specifically, accurately identify the whole growth cycle of $Z$. latifolia. Microstructure observations showed that the presence of $U$. esculenta could be clearly observed after gall formation but was absent after the TDF treatment. A total of 17,541 differentially expressed genes (DEGs) were identified, based on the transcriptome. According to gene ontology term and Kyoto Encyclopedia of Genes and Genomes pathway results, plant hormone signal transduction, and cell wall-loosening factors were all significantly enriched due to $U$. esculenta infecting $Z$. latifolia; relative expression levels of hormone-related genes were identified, of which downregulation of indole 3-acetic acid (IAA)-related DEGs was most pronounced in JB_D versus JB_B. The ultra-high performance liquid chromatography analysis revealed that IAA, zeatin+trans zeatin riboside, and gibberellin 3 were increased under $U$. esculenta infection. Based on our results, we proposed a hormone-cell wall loosening model to study the symbiotic mechanism of gall formation after $U$. esculenta infects Z. latifolia. Our study thus provides a new perspective for studying the physiological and molecular mechanisms of $U$. esculenta infection of $Z$. latifolia causing swollen gall
\end{abstract}

${ }^{\dagger}$ Corresponding author: S. Zhu; sdzhuaau@sina.cn

The raw RNA-seq data used in this study were deposited in the National Center for Biotechnology Information (NCBI) Sequence Read Archive (SRA) database, under the accession number SRP212030.

Funding: This work was supported by Anhui Province Key Research and Development Program (201904a06020057), Anhui Province Science and Technology Project (1304032040), Natural Science Foundation of Anhui Province (2008085MC80) and Anhui Provincial Education Department Natural Science Research Key Project (KJ2019A0188).

*The $e$-Xtra logo stands for "electronic extra" and indicates there are supplementary figures and tables published online.

The author(s) declare no conflict of interest.

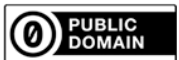

The author(s) have dedicated the work to the public domain under the Creative Commons CCO "No Rights Reserved" license by waiving all of his or her rights to the work worldwide under copyright law, including all related and neighboring rights, to the extent allowed by law. 2021 formations as well as a theoretical basis for enhancing future yields of cultivated $Z$. latifolia.

Keywords: gall formation, hormones, RNA-seq, symbiotic mechanism, Ustilago esculenta, Zizania latifolia

Zizania latifolia (Griseb.) Stapf, known as wild rice (Yang et al. 2014), is the only member of the wild Oryza sativa genus Zizania belonging to the Poaceae family that is native to Asia. In ancient China, Z. latifolia was one of the six most significant grain crops cultivated (Quan et al. 2009), being a perennial aquatic vegetable that can be grown in lakes and ponds, and which originated from China and southeastern Asia (Guo et al. 2007). This plant is an asexual aquatic vegetable with a unique flavor and texture that, due to the colonization of Ustilago esculenta, has since been cultivated in east and southeast Asia for more than 1,500 years (Chung and Tzeng 2004). The Z. latifolia rice plants are cultivated as a delicious and nutritious vegetable, containing sugar, protein, and minerals, along with providing some amino acids essential for the human body (Yan et al. 2018). Additionally, when used medicinally, this plant may also help the body relieve coughs, dampness, and heat and prevent poisoning induced by alcohol as well as possessing antiatherogenic effects (Han et al. 2013; Moghadasian et al. 2019).

In its long use as an aquatic vegetable, all its parts are edible, including the swelling gall, rhizomes, and grains. Yet, after centuries of evolution, its use as a grain has completely disappeared in China, but it continues to be cultivated for its swollen gall. Z. latifolia is usually parasitized by U. esculenta, which stimulates the swelling of the gall under suitable environmental conditions (Guo et al. 2015; Qingsen 2006). The success of the swelling gall formation depends on the degree of $U$. esculenta infection of $Z$. latifolia, whose cultivation globally has led to the evolution of diverse white varieties of this vegetable plant, driven primarily by the extent of $U$. esculenta colonization. This infection of $Z$. latifolia that stimulates swollen galls to form is called normal 'Jiaobai' in China (You et al. 2011). When the fungus infects the host plant, it causes the latter's expansion by enlarging the host cell size and number so that the growing formation swells into juicy galls; however, an $U$. esculenta infection can completely inhibit inflorescence growth and seed production in Z. latifolia (Thrower and Chan 1980). The internal tissues of formed edible galls, containing the cells expansion, give the appearance of a white gall whose inside is full of fungal hyphae; hence, the reason it is called 
"white Z. latifolia." Previous studies have shown that the symbiosis of Z. latifolia and U. esculenta not only produces edible gall but also improves host photosynthesis and growth (Yan et al. 2013). Through microscopic observation of the gall formation of $Z$. latifolia, it was found that the inside contained a large amount of hyphae and spores of $U$. esculenta (Li et al. 2019; Zhang et al. 2014). These plant-fungi results may be due to the outcome of a series of internal changes caused by the defense response of $Z$. latifolia.

Fungal infection and host defense reaction are primary events involved in plant-fungus interactions (Baker et al. 1997). These interactions typically produce a range of responses, such as disease symptoms, formation of specialized structures, and changes in biological and morphological reactions in host plant cells (Doehlemann et al. 2008; Hahn and Mendgen 2001). Many plants interact with a fungus in a beneficial direction, such as in rhizobium symbiosis (Sanders 2011), or in a harmful way, such as that well-documented for some fungal diseases and necrosis of crops (Dean et al. 2012). Many fungi must obtain nutrients from their environment, and, in both plant-fungus symbiosis and the majority of pathogenic fungi, they penetrate their host without damaging the plant cell walls or membranes. In our study, we focused on the positive side of endophytic fungi living in plants. Furthermore, in soybean, by adopting RNA-seq methods, it was found possible to confirm the gene co-networks underpinning the potential defense against the rust pathogen Phakopsora pachyrhizi (Hossain et al. 2018; Wang et al. 2017). During plant-fungus interactions, the infecting pathogens stimulate host cell responses, which are translated into phytohormone signals, leading to the production of metabolites and the expression of various key genes in host plants (Horst et al. 2008; Rabe et al. 2013).

Plant responses to swelling galls involve a complex network of signaling mechanisms, which coordinate to regulate many complex changes, including cell expansion and biochemical processes (Bhargava and Sawant 2013; Chaves et al. 2003), but whose responses could vary widely depending on plant species (Ahmad and Prasad 2012). Hormone regulation is known to have a central role in plant responses to swollen gall formations because the kinds and levels of hormones govern diverse ecophysiological processes in plants, for which the fast-acting signaling transduction pathway can induce marked changes and plant expansion (Wilkinson et al. 2012). Both indole 3-acetic acid (IAA) and cytoberberin (CTK) are cell-responsive hormones that play a crucial role in how plants sense the presence of swelling galls in them (Davies et al. 2005; Mizoi et al. 2012). In recent years, for many plant-fungi relationships, the stimulated symptoms will often reveal an imbalance of hormones, as seen in the swelling galls stimulated by microbes via production of IAA and CTK (Doonan and Sablowski 2010; Morrison et al. 2015). The formation of a swelling gall might have evolved from a process that mainly involved the ability of IAA or CTK to inhibit cellular defense responses (Gelvin 2003). This would be a typical interaction in which $U$. esculenta infects $Z$. latifolia and these interactions cause hormonal changes in the plant body, leading to gall formation and swelling expansion. For plant-fungi symbiosis, this hormone production tends to elicit changes in the root system that are often required to facilitate these ecological interactions (Hirsch et al. 1997). However, some pathogenic bacteria whose infection does not produce host cell deformations can also prompt the plant to generate and secrete phytohormones, indicating a likely role for such molecular mechanisms in biological processes other than cell deformation per se. In China and Southeast Asia, swollen galls are harvested as an aquatic vegetable, for placement on the dining table, it being edible and rich nutritious food. Interestingly, Z. latifolia is possibly the only cultivated food that arises from a plant-fungi interaction (Zhang et al. 2012). Even though many researchers have tried to survey plant-fungi interactions at both their hormonal and physiological levels, significant differences in host plant responses to U. esculenta as compared with other fungi have yet to be comprehensively investigated or interpreted (Yan et al. 2013; Zhang et al. 2014). Therefore, the symbiosis mechanisms responsible for the formation of plantfungus swollen galls and their further growth remain to be uncovered, which, if done, could explain how the phytohormone response arises and regulates this process.

Triadimefon (TDF) (CAS: 43121-43-3) is widely used in agriculture and medicine as a fungicide, as it can prevent fungal colonization and inhibit the formation of galls and hyphae. Exogenous spraying of TDF has been studied in many fields for a long time. Such studies report that spraying TDF at the eightleaf stage of $Z$. latifolia plants can inhibit water-driven swelling of the gall and, within a certain range, it has a discernible dose effect. By observing stem tissue slices, it was recently found that TDF can clearly inhibit $U$. esculenta growth and formation and that $Z$. latifolia gall formation is closely related to the distribution of $U$. esculenta in the host ( $\mathrm{Li} 2016)$. To further search for related genes participating in the $U$. esculenta infection of $Z$. latifolia and causing gall formations that are closely related, transcriptome analysis is warranted. Previous studies have revealed that, when Z. latifolia plants were sprayed with TDF, swollen gall formations are less likely to occur, while the hereditary shape of male Z. latifolia can differ from normal Z. latifolia (Yan et al. 2013). Treatment with TDF was able to solve the problem of genetic variation. In preliminary work for this study, we sprayed it directly onto Z. latifolia, and these results showed no U. esculentacolonized plants and no swelling gall formation in them. Thus, using TDF may help in the necessary further investigations of $Z$. latifolia gall information at the molecular level.

Currently, the study of Z. latifolia molecular biology is still in the early stage. Transcriptome methods have been successfully used to explore a wide variety of plant development processes and pathogen-infection interactions (Guo et al. 2015). Although some researchers (Guo et al. 2015) have tried to explain the detailed signal pathways related to the gall-formation mechanism in Z. latifolia, many questions remain unanswered. In this study, we analyzed the swelling formation of gall in Z. latifolia infected with $U$. esculenta. Ultrastructure, paraffin sections, and scanning observations were all made to look for the distribution and abundance of $U$. esculenta. TDF was used to eliminate the effect of $U$. esculenta infection on hosts, and transcriptome sequencing was carried out before and after in normal (control) and TDF-treated plants, so we could analyze the transcriptome spectrum of differentially expressed genes (DEGs) and detect the full gene expression spectrum. In this way, these study results may provide new, timely insights into the gall formation mechanism of $Z$. latifolia.

\section{RESULTS}

\section{Morphological observations and period identification on the whole life cycle of $\boldsymbol{Z}$. latifolia.}

The phases of $Z$. latifolia development and growth had not yet been determined but discerning these was crucial for using an accurate period in the experiment and its TDF treatment for subsequently experiments. So, we observed and photographed the $Z$. latifolia at 10-day intervals starting 20 days after transplanting. The photos were divided into a front view, cross section, and vertical section (Fig. 1). In the 150 days after setting, we took photos every three days; a total of 27 groups of photos were taken in the entire process. The before gall formation period was divided as follows: i) three leaves (including three leaves) and 28 days after transplanting; ii) the fifth-leaf stage, 79 days after the transplanting of $Z$. latifolia; iii) the 
seventh-leaf stage, 110 days after the determination of Z. latifolia; and iv) 153 days after transplanting $Z$. latifolia. Periods after gall formation were i) 156 days after transplanting, ii) 159 days after transplanting, iii) 162 days after transplanting, iv) 165 days after transplanting, and v) 168 days after transplanting. The observation of the periods laid a foundation for further before and after gall formation study of $Z$. latifolia.

\section{Microscopic observations of $\boldsymbol{U}$. esculenta in different} sections reveal its invasion process of $Z$. latifolia.

In the normal growth of $Z$. latifolia, the formation of swelling galls was evident (Fig. 2A). However, treating Z. latifolia with TDF prevented the formations of swollen galls (Fig. 2B). We observed the samples of before and after gall formation in untreated (CK) and TDF plants. It was found that no swelling gall had formed early in plant development but in the late period of the $\mathrm{CK}$ group, the Z. latifolia stem continued to swell in size (Fig. 2C). Optical microscopy (aniline blue staining) showed that the hyphae could only be observed after gall formation in CK, based on examinations of ordinary sections and paraffin sections (Fig. 2D and E). The infection processes of $U$. esculenta in the gall of CK and TDF treatment were also observed using scanning electron and ultrastructure microscopy methods (Fig. 2F and G). As shown in Figure $2 \mathrm{~F}$ and $\mathrm{G}$, no difference was apparent between JB_A and JB_C before any swelling galls formed. In JB_B, however, we find many round spores. These sporophytes were clustered together and attached to the cell wall. The fungal cell wall can be clearly seen in the ultrastructure images, closely attached to the plant cell wall, where it may communicate with the plant body.

\section{Analysis of different hormone responses to $U$. esculenta infection.}

To detect the levels of hormonal response to U. esculenta infection, ultra-high performance liquid chromatography (UHPLC) was used to measure IAA, ABA (abscisic acid), Z+ZR (zeatin and trans zeatin riboside), and $\mathrm{GA}_{3}$ (gibberellin 3) contents (Fig. 3) in Z. latifolia before and after gall formation in both CK and TDF groups. As Figure 3A shows, the $\mathrm{Z}$ content was high in JB-B, but it decreased sharply, by $8.21 \%$, from JB-B to JB-D, with no significant change from JB-A to JB-C. To our surprise, we also found that $\mathrm{GA}_{3}$ and IAA contents exceeded the others, suggesting the contents of IAA, ABA, and $\mathrm{GA}_{3}$ may be driven by the $U$. esculenta infection-plant symbiosis, which could have stimulated the plant to produce a large amount of growth hormones to cause the characteristic swollen gall formation. Both Z and ZR belong to cytokine (CTK) and are usually considered as CTK together. We found that the content of JB-B in these ratios was always higher than that of JB-D, while there was no significant change in JB-A and JB-C. According to Figure 3 , we found that there was a significant difference in IAA, $\mathrm{GA}_{3}$, and Z+ZR in JB-B versus JB-D. These results indicated that $U$. esculenta infection of $Z$. latifolia could stimulate the host to produce a large amount of the growth-promoting hormones that fuel gall formation and expansion.

\section{Identification of DEGs by RNA-seq.}

To study the differences in RNA-seq between $U$. esculentainfected and noninfected Z. latifolia plants before and after gall formation, three biological replicates were used per group, for a total of 12 complementary DNA (cDNA) samples (Table 1; Supplementary Table S1), a total of $84.42 \mathrm{~Gb}$ of clean reads was generated. A total of 17,541 DEGs were optimized by known structures (Fig. 4A). The significant Pearson correlations (Supplementary Fig. S1) obtained between the FPKM (fragments per kilobase per million reads) distributions of biological replicates of all samples confirmed the high reproducibility of our sequencing data. As Figure 4 shows, there were respectively 3,122, 2,672, 6,704, and 5,042 DEGs, identified by the DESeq R package, based on FPKM data in JB_B versus JB_A, JB_D versus JB_B, JB_D versus $\mathrm{JB} \_\mathrm{C}$, and $\mathrm{JB} \_\mathrm{C}$ versus $\mathrm{JB} \_\mathrm{A}$, respectively. Venn and Upset plot diagrams were made based on the up- and downregulation data in Figure 4C and D, from which it emerged that the four sample groups did not share all upregulation or downregulation DEGs in common, suggesting that the gall before and after formation and TDF reached the expected effect in $Z$. latifolia.

Gene ontology (GO) term and Kyoto Encyclopedia of Genes and Genomes (KEGG) enrichment analyses for all DEGs.

To identify the similarities and differences between the pairings of JB_B and JB_A and JB_D and JB_B in transcriptomes

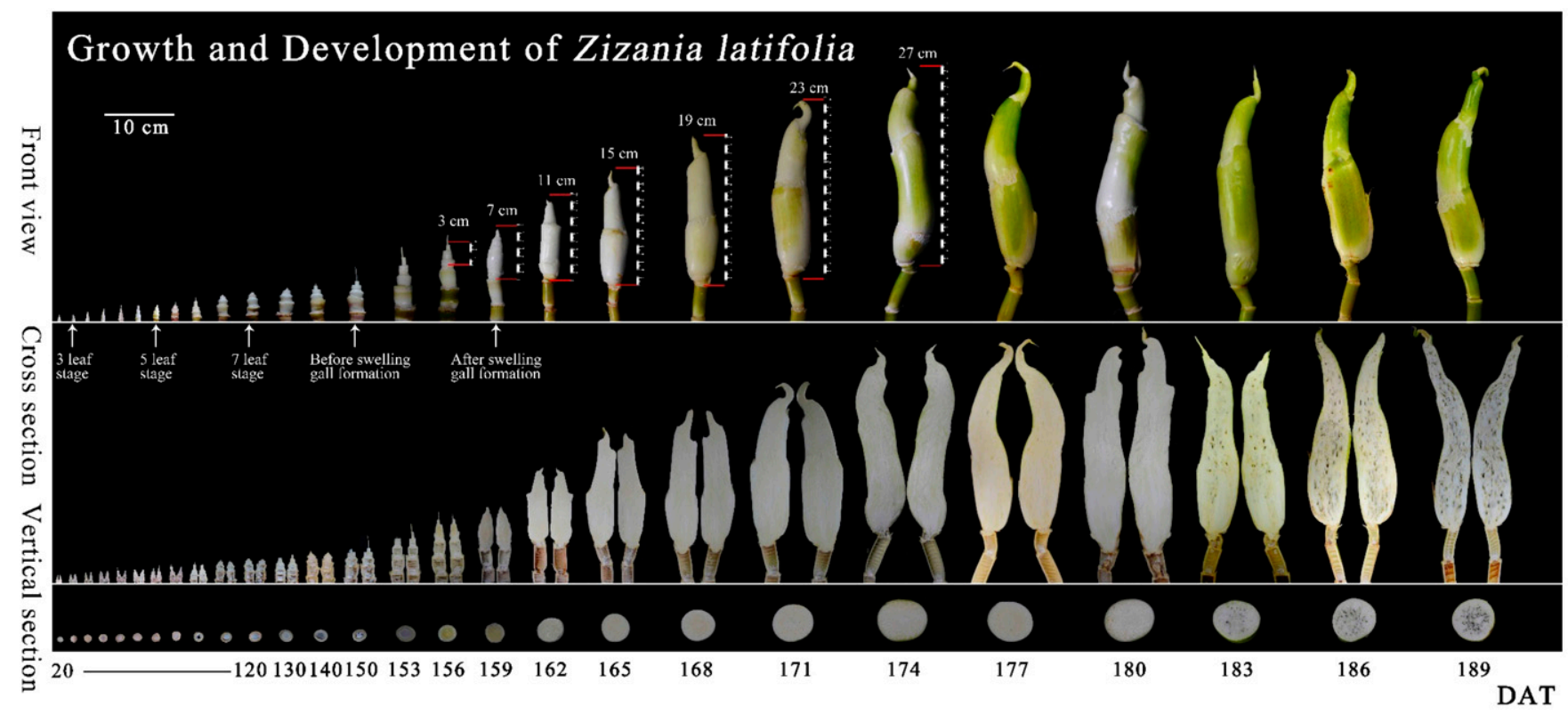

Fig. 1. Observations on the growth and development period of Zizania latifolia plants. We confirmed the before and after morphological changes in swelling gall formation of $Z$. latifolia. DAT = days after transplanting. 
A
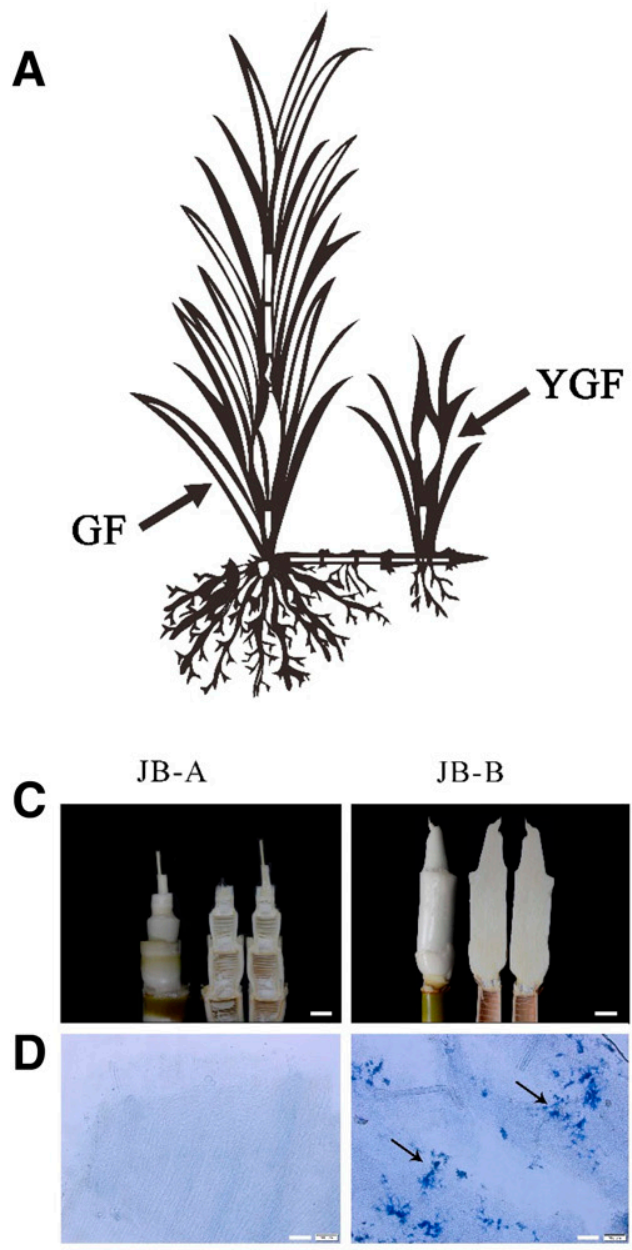

E

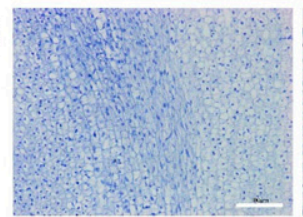

F

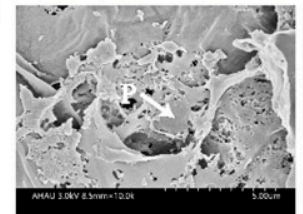

G

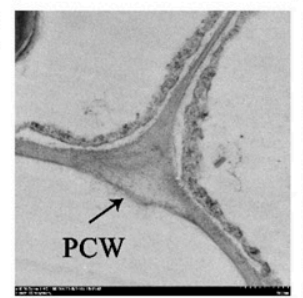

JB-B
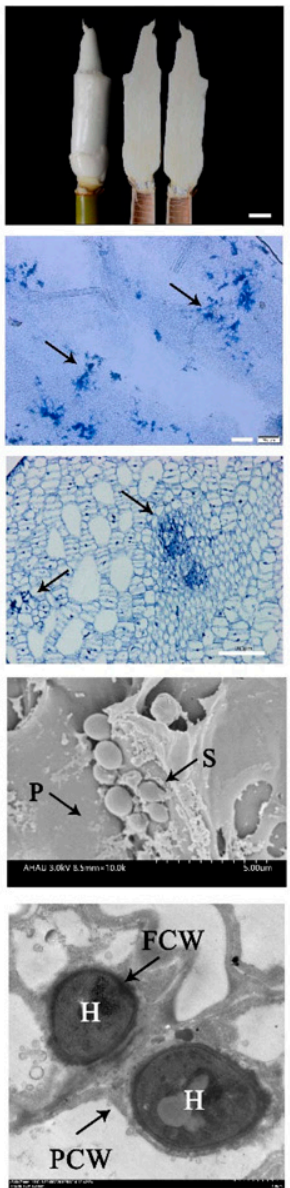

B

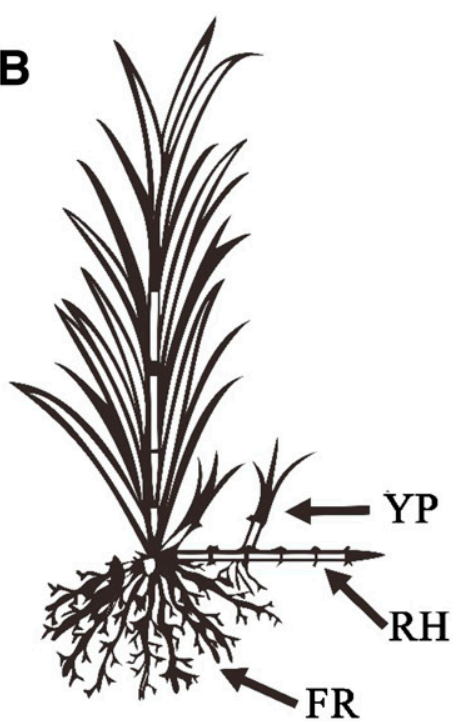

JB-C

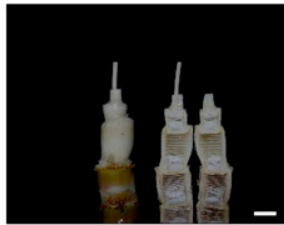

JB-D
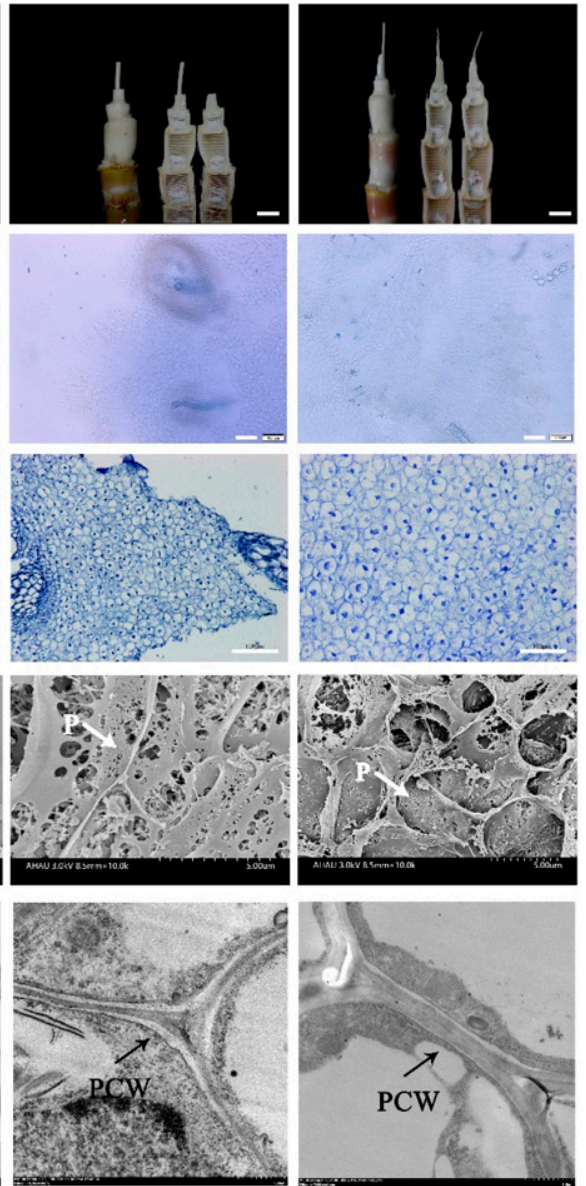
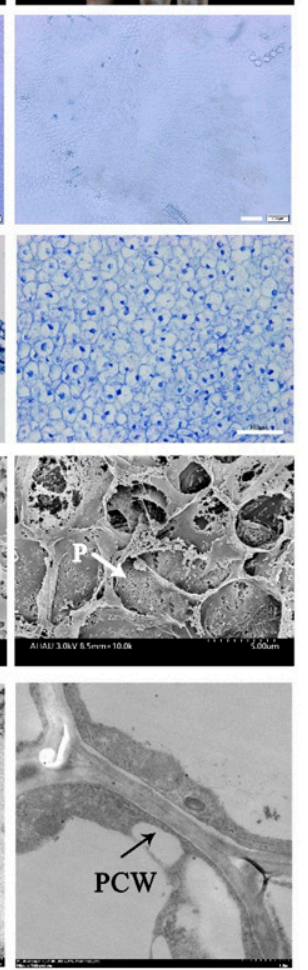

Fig. 2. Sample pictures, ordinary sections, and paraffin sections were observed in different samples. A, Normally grown Zizania latifolia with swollen gall formations. Arrows point to swelling gall formation (GF) and young gall formation (YGF). B, Triadimefon (TDF)-treated Z. latifolia with no formation of swollen gall. Arrows point to gall fibrous root (FR), rhizome (RH), and young plants (YP). C, JB-A shows control (CK), before swollen gall formation in Z. latifolia; JB-B shows CK, after swollen gall formation in Z. latifolia; JB-C shows TDF treatment, before swollen gall formation in Z. latifolia; JB-D shows TDF treatment after swollen gall formation in Z. latifolia. Bar $=1 \mathrm{~cm}$. D, The Ustilago esculenta was observed by aniline blue stain in different samples. The arrows indicate the presence of clustered $U$. esculenta. Bar $=100 \mu \mathrm{m}$. E, Paraffin sectioning, showing the clear observation of cell size and fungus using optical microscope. Bar $=100 \mu \mathrm{m}$. F, Different samples were observed by scanning electron microscope. P points to the plant, $\mathrm{S}$ to the sporophore of $U$. esculenta . Bar $=$ $5 \mu \mathrm{m}$. G, Transmission electron microscopy images from before and after swollen gall formations of $Z$. latifolia . PCW $=$ plant cell wall, $\mathrm{H}=$ hyphae, FCW $=$ fungal cell wall. Bar $=500 \mathrm{~nm}$ (panels 1 and 3) and $1 \mu \mathrm{m}$ (panels 2 and 4). 
under U. esculenta infection, their corresponding DEGs were subjected to GO classification and KEGG functional enrichment analysis. In comparing the distribution of these DEGs and all top-10 ranked genes, they were allocated to three categories: biological process, cellular component, and molecular function (Supplementary Fig. S2; Supplementary Table S3). The most common GO terms obtained were 'planttype cell wall,' 'DNA-binding transcription factor activity,' and 'extracellular region'. Therefore, the corresponding genes of these important terms might play a central role in defending against $U$. esculenta infection (or perhaps tolerating it). Many different genes associated with cell wall-loosening factors (e.g., EXPA, EXPB, XTHs, FLAs) had also been altered. The KEGG enriched top-20 pathways of these two pairings are shown in Figure 5B and D. For plant hormone signal transduction (Ko04075), 48 and 37 DEGs were identified in the JB_B versus JB_A and JB_D versus JB_B pairings, respectively (Supplementary Fig. S5; Supplementary Table S4).
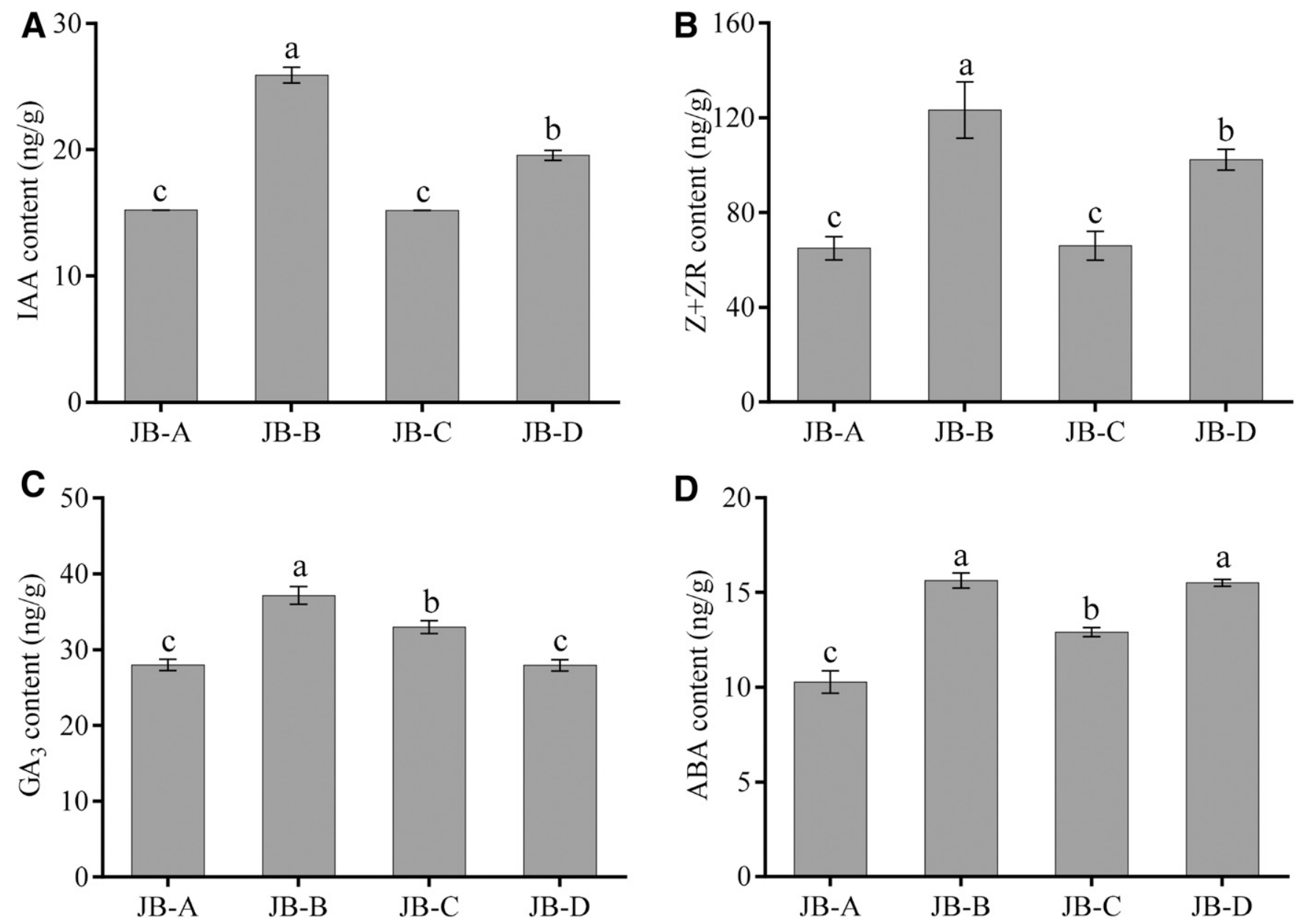

Fig. 3. Content of hormones in Zizania latifolia plants before and after gall formation under the untreated and triadimefon (TDF) treatments. The hormones determined were A, indole 3-acetic acid (IAA), B, Z+ZR (zeatin and trans zeatin riboside), C, GA 3 (gibberellin 3). and D, ABA (abscisic acid). Values represent the mean \pm standard error $(n=3)$. Lower case letters above bars indicate significant differences at $P<0.05$, according to Duncan's multiple range tests.

Table 1. Quality assessment of raw RNA-seq data

\begin{tabular}{|c|c|c|c|c|c|}
\hline Sample & Clean_reads (Million) & Clean_bases (Gb) & Valid_bases (\%) & Q30 (\%) & GC $(\%)$ \\
\hline Sample_JB_A1 & 48.05 & 7.02 & $95.46 \%$ & $95.80 \%$ & $52.92 \%$ \\
\hline Sample_JB_A2 & 48.13 & 7.03 & $95.54 \%$ & $96.03 \%$ & $52.61 \%$ \\
\hline Sample_JB_A3 & 47.97 & 6.99 & $95.03 \%$ & $95.45 \%$ & $52.86 \%$ \\
\hline Sample_JB_B1 & 48.00 & 7.00 & $95.33 \%$ & $95.70 \%$ & $52.80 \%$ \\
\hline Sample_JB_B2 & 48.87 & 7.11 & $95.29 \%$ & $96.06 \%$ & $52.59 \%$ \\
\hline Sample_JB_B3 & 48.57 & 7.07 & $95.27 \%$ & $95.94 \%$ & $52.70 \%$ \\
\hline Sample_JB_C1 & 47.97 & 6.98 & $95.04 \%$ & $95.75 \%$ & $53.52 \%$ \\
\hline Sample_JB_C2 & 48.77 & 7.10 & $95.22 \%$ & $95.97 \%$ & $53.18 \%$ \\
\hline Sample_JB_C3 & 48.25 & 7.04 & $95.39 \%$ & $95.91 \%$ & $53.00 \%$ \\
\hline Sample_JB_D1 & 48.67 & 7.06 & $94.86 \%$ & $96.09 \%$ & $52.17 \%$ \\
\hline Sample_JB_D2 & 48.04 & 6.97 & $94.61 \%$ & $95.72 \%$ & $52.16 \%$ \\
\hline Sample_JB_D3 & 48.72 & 7.05 & $94.42 \%$ & $95.75 \%$ & $52.23 \%$ \\
\hline
\end{tabular}


The divergent expression patterns of hormone metabolismrelated DEGs between the CK and TDF groups suggest that plant hormones play a key role in governing responses to $U$. esculenta infection in Z. latifolia hosts.

Regulation overview, abiotic stress enrichment, and transcription factor (TF) analyses of all DEGs.

Regulatory pathways in JB_D versus JB_B were studied with the MapMan tool for annotation analyses (Fig. 6; Supplementary Table S2), and a regulation overview enrichment of DEGs that were up-regulated and functionally enriched in TFs included the homeobox TF family and receptor kinases (Fig. 6A). Some pathways, including those of 'protein modification,' and 'calcium regulation,' were also up-regulated or down-regulated (Supplementary Table S2 A) in comparing the response of JB_D versus JB_B. Most DEGs linked to plant hormones were associated with upregulation of cytokinin, ethylene (ETH), and IAA, with only gene expression for ABA undergoing a downregulation (Fig. 6A; Supplementary Fig. S3; Supplementary Table S3A). Through abiotic stress analysis (Fig. 6B), the relevant genes could be divided into signal transduction, pathogenesis-related (PR) protein, TFs, hormones ABA, salicylic acid (SA), jasmonic acid (JA), and ETH, thus further supporting our inference of the crucial participation of these pathways in the symbiotic environment, for the regulated sensing and promotion of responses to $U$. esculenta infection in Z. latifolia. A more detailed list of all the DEGs corresponding to MapMan functional categories is provided (Supplementary Table S3). The TFs involved were primary regulators of DEGs expression and perform significant functions in the transcriptional symbiosis of plant-fungi genes after infection by $U$. esculenta. Going further, we described TFs in detail and found that homologous box (HB) and basic helix-loop-helix (bHLH) had more differentially upregulated expression (Fig. 6C). Many TFs were significantly altered, as inferred by changes in expression of their corresponding genes. Among them, the auxin (AUX) response factor (ARF) family is an important participant, for which the $A R F 10$ and $A R F 21$ genes were respectively down-regulated by 4.45 -fold and 5.01 -fold (Fig. 7; Supplementary Table S6) in JB_D versus JB_B. We show that the expression levels of 308 putative TF-relative genes can be divided into 42 TF families in the JB_D versus JB_B pairing (Supplementary Table S3C). These TFs might be involved in hormone regulation, cell differentiation, and expansion, as well as expression patterns in different organisms after they undergo $U$. esculenta infection. In sum, these TFs seem to be linked to stimulating the response in Z. latifolia to gall formation, according to the annotation information. In the
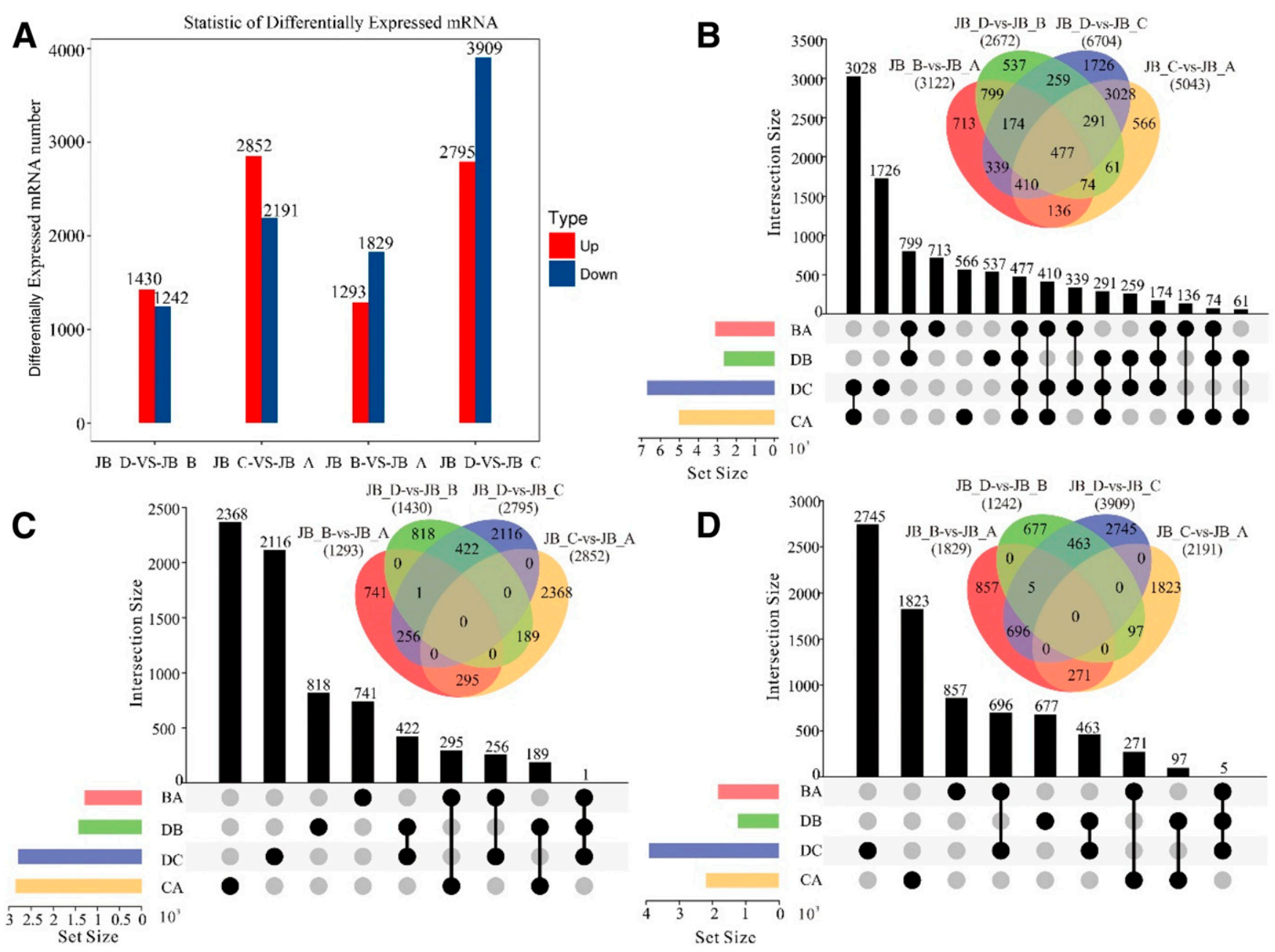

Set Size

Fig. 4. Numerical values of differentially expressed gene (DEG) analysis. A, For the RNA-seq data set, the number of all genes and the number of up- or downregulated genes in four comparison groups. The numbers of upregulated genes are in red, the downregulated genes in blue. B, Venn and Upset plot diagrams of the DEGs in the different group comparisons. The numbers indicate unique and common DEGs in three replicates for the different comparisons. $\mathbf{C}$ and $\mathbf{D}$, Venn and Upset plot diagrams of the upregulated and downregulated genes in different comparisons. 
comparison of JB_D versus JB B , the majority of DEGs were down-regulated and functioned in the enrichment of TFs including ARF, bHLH, receptor kinases, and HB. Receptor kinases are involved in a variety of plant responses, including hormone sensing and reacting to pathogens, making them key players in defense responses.

\section{Changes in expression of plant hormone signal transduction pathway-related genes after $U$. esculenta infection.}

The expression of genes related to plant hormone signal transduction indicated meaningfully dynamic changes that occurred during the $U$. esculenta infection process. These DEGs were related to IAA, CTK, GA, ABA, ETH, JA, and SA hormones (Fig. 7; Table 2) and were observed as follows in the four pairing: 37 for JB_D versus JB_B, with all DEGs associated with plant hormone signal transduction (Supplementary Fig. S5).

The IAA-related pathways had the greatest response to plant phytoplasma infections. In the IAA signal transduction pathway, nine genes were up-regulated in JB_D versus JB_B, including four AUX-responsive proteins (AUX/IAA), three AUX transporter-like proteins (AUX influx carrier family), one AUX-responsive GH3 gene family (GH3), and one ARF. By contrast, three AUX/IAA (Zlat_10001127, Zlat_10020059, Zlat_10033997) proteins, and an AUX transporter-like protein (AUX1, Zlat_10014750) were respectively down-regulated by $-1.26,-1.11,-1.52$, and -2.29 -fold, in the JB_D versus JB_B pairing. Most of the downregulated expression levels indicated that IAA signaling was obviously induced by U. esculenta infection. We suspect that AUX may result from gall formations caused by the growth substances produced by the infection of $U$. esculenta. In the CTK-signaling transduction pathway, two DEGs (Zlat_10010976, Zlat_10018529) were found upregulated (1.31-fold and 1.22-fold, respectively), including the two-component response regulator ARR (B-ARR). In the GA-signaling pathway, an encoding TF (phytochrome-interacting factor 4, Zlat_10039510) was significantly down-regulated for JB_D versus JB_B. Interestingly, ten ABA-related DEGs were up-regulated with respect to the JB_D versus JB_B signal transduction pathways (Fig. 7). The ABA receptor protein phosphatase 2C (PP2C), serine/threonine-protein kinase (SnRK2), and ABA-insensitive (ABF) TF family each had upregulated DEGs in the signal transduction pathway and they participate in the negative regulation of ABA signaling. For example, PP2C (Zlat_10022265) was up-regulated by 4.96-fold in JB_D versus JB_B. Upregulation of these key DEGs in the ABA pathway indicated that ABA expression might be increased
A

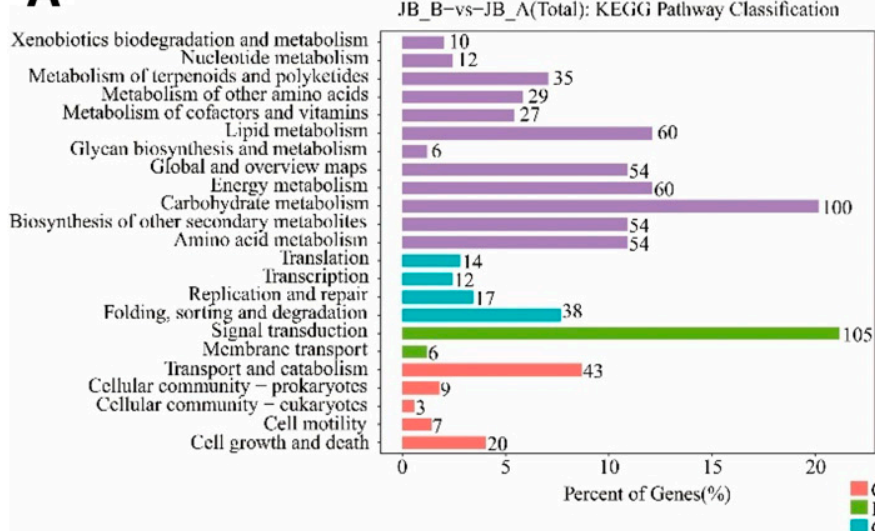

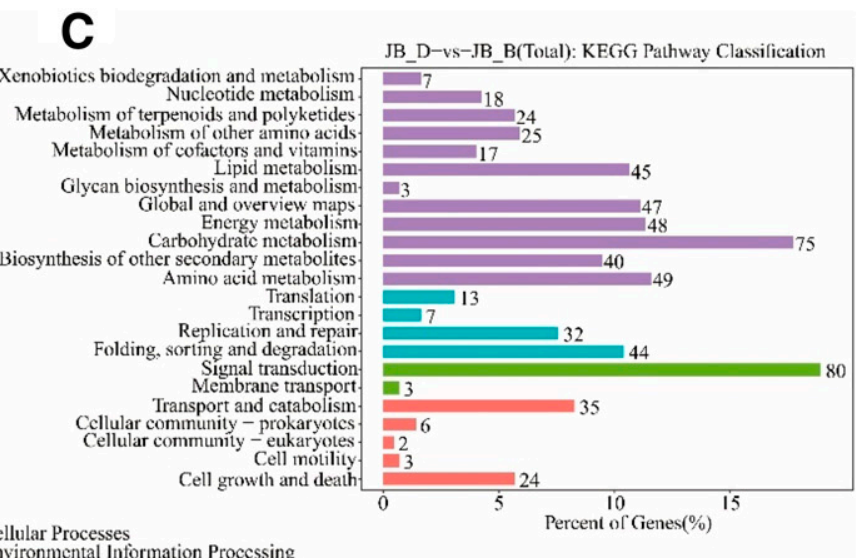

B

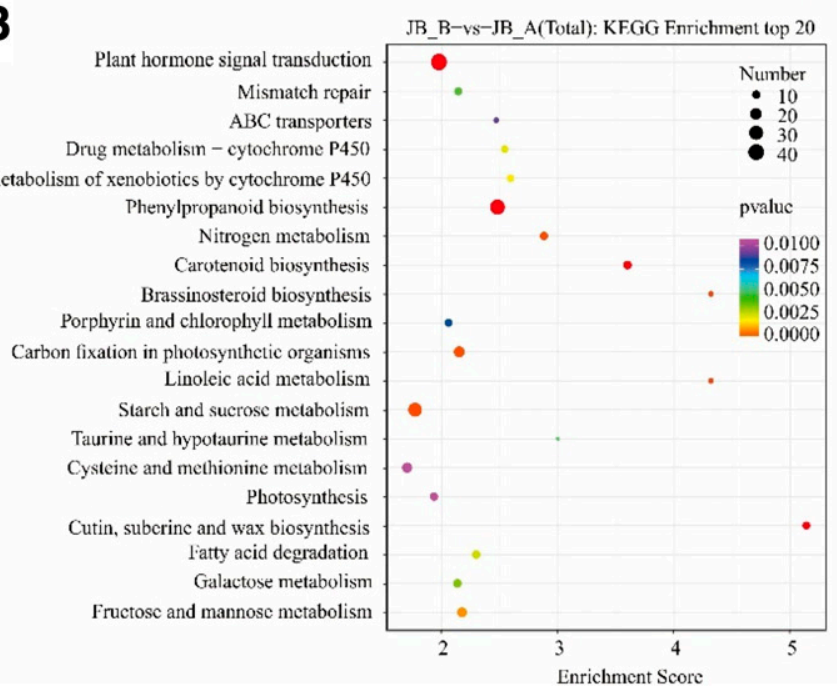

D

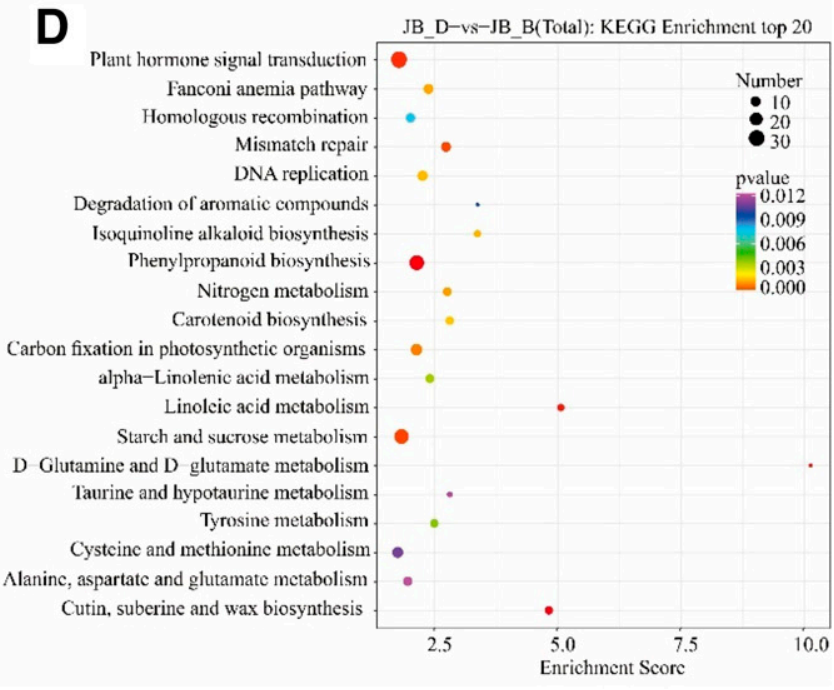

Fig. 5. Classification and enrichment analyses of Kyoto Encyclopedia of Genes and Genomes (KEGG) pathways. A and C, The histograms of the KEGG pathways classification for the differentially expressed genes (DEGs) of JB_B versus JB_A and JB_D versus JB_B, respectively. B and D, Bubble diagrams of top 20 enriched KEGG pathways for the DEGs of JB_B versus JB_A and JB_D versus JB_B, respectively. Dot size represents the number of genes, and its color the q-value range. 
after the TDF treatment causes cell senescence. Two genes involved in JA signaling were also down-regulated (JARI, JAamino synthetase) for JB_D versus JB_B. From seven SArelated genes identified here, six were related to the transduction factor TGA and one to the regulatory nonexpresser of PR genes 1 $(N P R 1)$. Generally, most of the DEGs were up-regulated. A few SA-related DEGs were nonetheless down-regulated, such as one associated with the regulatory protein NPR1 genes (upregulated
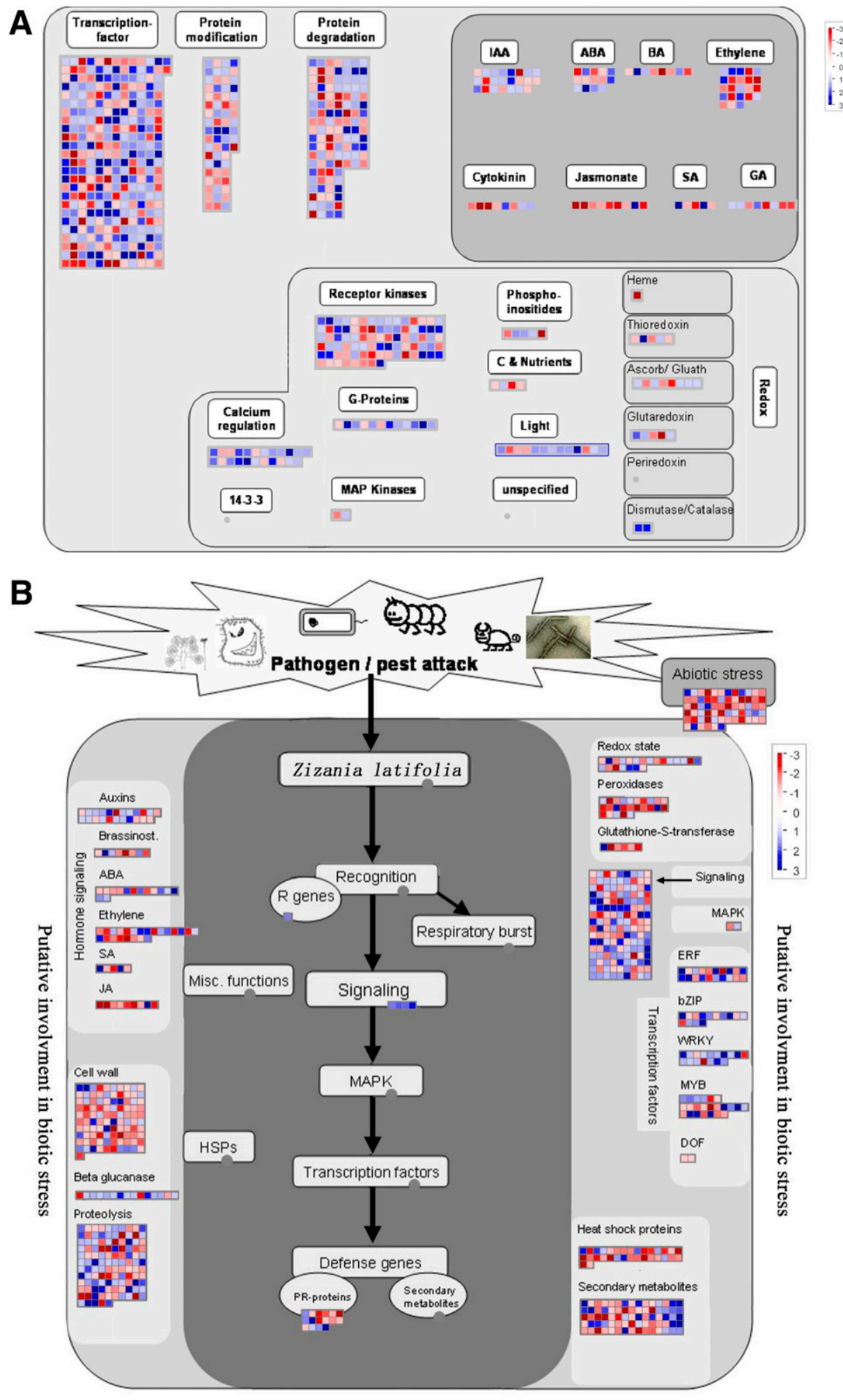

(Continued)

Fig. 6. Regulation overview of differentially expressed genes (DEGs) and transcriptional responses, visualized using Mapman software, for JB_D versus JB_B A, Regulation overview of enrichment among DEGs comparing untreated (CK) and triadimefon (TDF) treatments after swollen gall formation in Zizania latifolia plants. B, Biotic stress enrichment among the DEGs. C, Main transcription factor family enrichment pathway among the DEGs. Transcriptional changes occurring after gall formation in Z. latifolia groups of CK and TDF. Genes in blue or red correspond to those significantly upregulated or downregulated in Ustilago esculenta-infected gall samples relative to DEGs. Individual genes are represented by small squares. The vertical color scale displays the transformed $\log _{2}$-fold changes. 
by 2.16 -fold). In sum, most of the genes involved in IAA, CTK, and SA underwent downregulation in their expression, corresponding to downregulated hormone contents after U. esculenta infection. Conversely, most of the gene expression patterns involving SA, JA, and ABA metabolism and signal transduction were up-regulated by $U$. esculenta infection of in Z. latifolia (Fig. 7; Table 2).

Expression of plant hormone biosynthesis genes and their quantification by quantitative real time PCR (qRT-PCR).

A set of 25 DEGs (Fig. 8) were selected for real time qPCR analysis to confirm their functioning in the Z. latifolia response to $U$. esculenta infection. Comparing the transcriptome data with qRT-PCR results yielded a relatively strong correlation $\left(R^{2}=0.9723\right)$ (Supplementary Fig. S4), thus validating the robustness of the RNA-seq analysis in this study. Of the candidate DEGs, we chose 25 related to hormones, 10 relatead to IAA, four to CTK, three to ABA, three to SA, three to JA, and two to GA. Among these 25 DEGs, the upregulation of 20 and the downregulation of five were confirmed by the qRT-PCR in JB_D versus JB_B, which agreed with the RNA-seq analysis (Fig. 8).

The $A R F$ genes are central components of plant AUX signal transduction. To better understand how IAA and CTK may participate in and affect swollen gall formations, we identified genes encoding IAA and CTK signal transduction at the host gall tip and regulated their expression during gall formation. These candidates included 10 IAA genes (ZLIAA23, ZLIAA2, Os11g0169200, ZLSAUR39, ZLPLC2, ZLYUC11, ZLIAA9, ZLIAA10, ZLARF21, ZLIAA50) and four CTK (ZLCKX8,
ZLBPA 1, ZLRR1, ZLRR4) hormone-related genes. We detected that ZLIAA23, ZLIAA2, Os11g0169200, ZLSAUR39, ZLPLC2, ZLYUC11, ZLIAA10, ZLARF21, and ZLIAA50 were not obviously affected by $U$. esculenta infection before gall formation. Yet, during or after $U$. esculenta infection, their expression levels were significantly induced (Fig. 8). Importantly, the expression of SAUR39 and PLC2 (Fig. 8; Supplementary Table S6) were down-regulated by the greatest magnitude (21.06- and 10.42-fold, respectively). Similarly, ZLCKX8, ZLBPA1, and $Z L R R 4$ were expressed consistently in the $C K$ group before gall formation, but, with $U$. esculentai present, its expression level was obviously up-regulated in Z. latifolia.

\section{Interaction network constructed based on the plant hormones and cell wall-relative genes.}

We selected many of those genes found related to plant hormones and cell walls to construct their interaction network (Fig. 9). In this figure, the outermost layer is related to cell-wall genes. Interestingly, we found that most of the genes were, in fact, related to cell wall softening-factor genes, while those most clearly expressed internally are AUX-related genes (Fig. 9A), a relationship that was particularly strong. Meanwhile, we selected genes for GO and KEGG annotation analysis. Most of them are located in plant hormone signal transduction and cell wall biogenesis, according to the KEGG annotation; in the GO analysis, we found that most of the genes are in the nucleus that controls gene expression (Fig. 9B).

We discerned significant differences in the expression of related DEGs in the cell walls of $U$. esculenta-infected Z. latifolia (Fig. 6B). Among these DEGS, the $\beta$-expansin gene $(E X P B 11)$ had the strongest downregulation in JB_D versus

Fig. 6. (Continued from previous page)

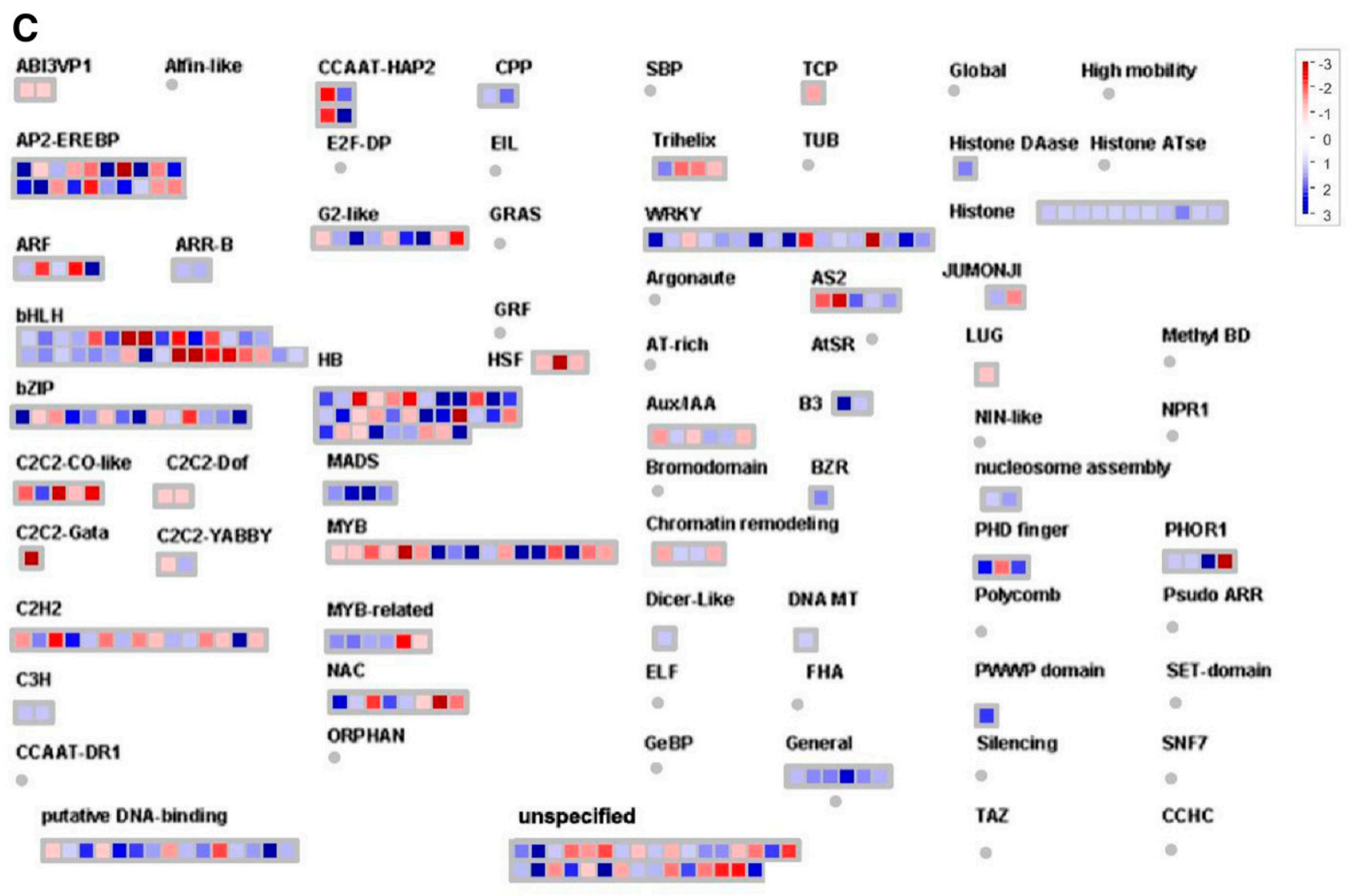

176 / Molecular Plant-Microbe Interactions 
JB_B, but many similar genes were also down-regulated (EXPB3, EXPB6, EXPB2, and EXPA8, among others). Meanwhile, xyloglucan endotransglu-cosylase/hydrolase (XTH)related genes were down-regulated, namely XTH32, XTH28, $X T H 31$, and XTH8. Fasciclin-like arabinogalactan (FLA) proteins are an important component of cell-wall genes (Supplementary Table S7), for which large numbers of FLA-type genes (i.e., FLA1, FLA2, and others) were down-regulated in the transcriptome (Supplementary Fig. S2). AUX is considered pivotal to controlling the invasion of $U$. esculenta, given that AUX/IAA and ARF were expressed most strongly in the gene expression pattern analysis, with AUX1 upstream initiating AUX expression only after $U$. esculenta infection. During infection by $U$. esculenta, CTK, a hormone capable of promoting cell proliferation, can act as the second messenger of IAA signaling while also promoting gall expansion (Fig. 9).
Moreover, IAA transport enhances levels of GA by promoting its TF and upstream genes. Therefore, positive or negative interactions between IAA and CTKs, GA, ABA, JA, and SA can be observed during the gall formation process of $U$. esculenta infection, which implicates IAA as the cornerstone of this regulatory network (Fig. 9A). In this way, we surmised that cell wall-loosening factors clustered around the hormones, with AUX positioned at the center and other hormones functioning to regulate the expansion of the gall in $U$. esculenta-infected Z. latifolia.

\section{DISCUSSION}

Many fungi interact with their plant hosts in beneficial ways, such as rhizobium symbiosis (Sanders 2011), or they interact with plant hosts in harmful ways, such as in the case of fungal

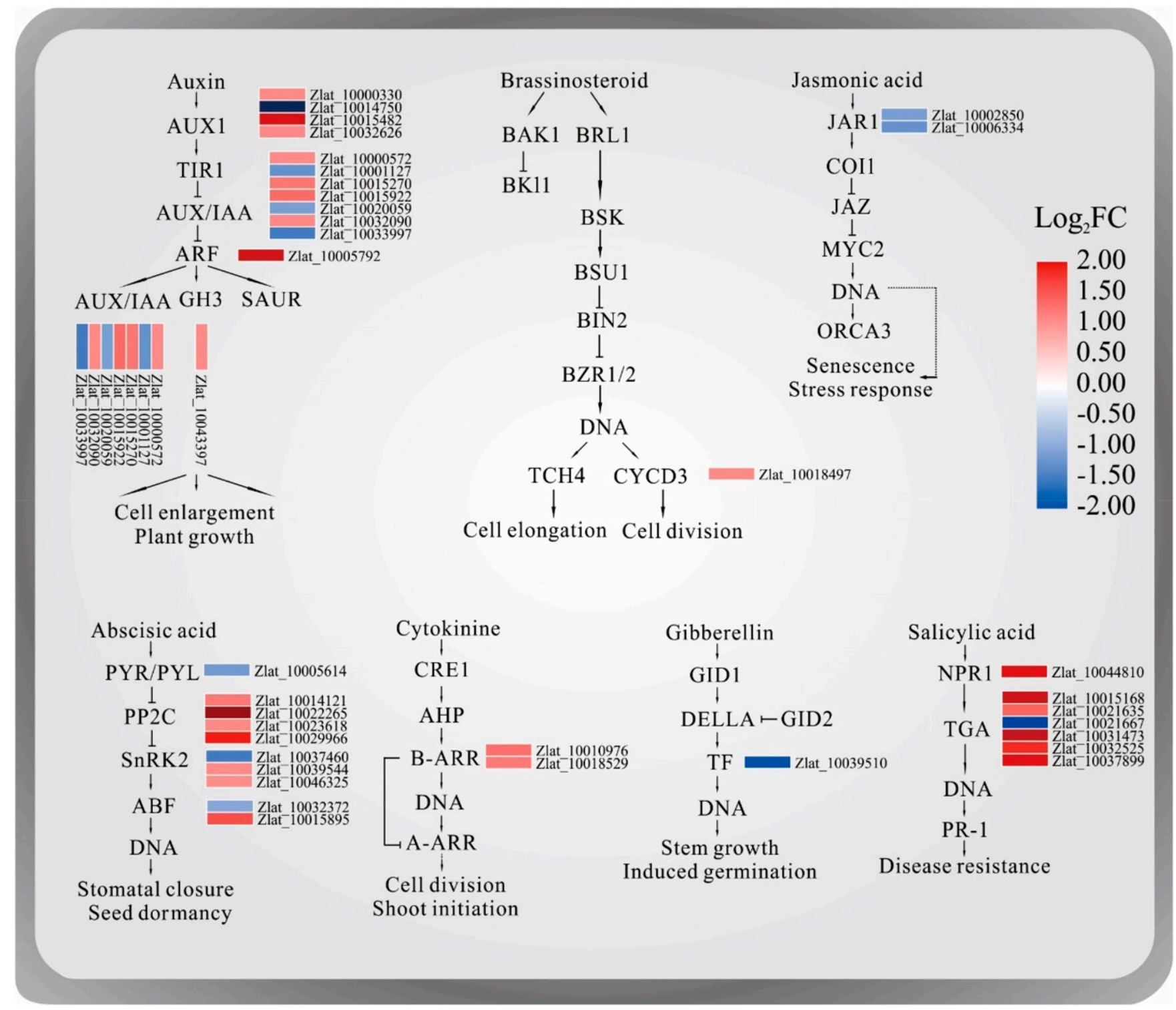

Fig. 7. Expression of genes involved in hormones for JB_D versus JB_B. Red indicates the upregulated genes, blue the downregulated genes, by heatmap. Their values were $\log _{2}$-fold change. AUX1 = auxin transporter-like protein; TIR1 = protein transport inhibitor response $1 ;$ AUX/IAA $=$ AUX responsive protein indole 3-acetic acid; ARF = AUX response factor; GH3 = AUX-responsive GH3 gene family GH3; SAUR = AUX-responsive protein SAUR; CYCD3 = cyclin D3, plant; JAR1 = jasmonic acid-amido synthetase JAR1; COI1 = coronatine-insensitive protein 1; JAZ = protein TIFY; MYC2 = transcription factor MYC; $\mathrm{PYR} / \mathrm{PYL}=$ abscisic acid receptor PYR/PYL family; PP2C $=$ protein phosphatase $2 \mathrm{C}$; TF $=$ phytochrome-interacting factor 4 ; SnRK2 = serine/threonineprotein kinase SRK2; $\mathrm{ABF}=$ abscisic acid-insensitive; $\mathrm{AHP}=$ histidine-containing phosphotransfer protein; $\mathrm{A} / \mathrm{B}$-ARR $=$ two-component response regulator ARR; GID1 = gibberellin receptor GID1; GID2 = gibberellin receptor GID2; DELLA = DELLA protein RG; NPR1 = nonexpresser of PR genes 1; TGA = transcription factor TGA; PR-1 = pathogenesis-related protein 1. 
Table 2. Identities and analysis of differentially expressed genes enriched in plant hormone signal transaction pathways of JB_D vs. JB_B

\begin{tabular}{|c|c|c|c|c|c|c|}
\hline $\begin{array}{l}\text { Hormone category/ } \\
\text { gene ID }\end{array}$ & $\log _{2}$ fold change & $P$ value & Up/down & Gene symbol & Pathway position & Description \\
\hline \multicolumn{7}{|l|}{ Salicylic acid } \\
\hline Zlat_10015168 & 3.038 & 0.036601284 & Up & TGAL5 & TGA & $\begin{array}{l}\text { Os05g0443900, partial (Oryza sativa } \\
\text { japonica group) }\end{array}$ \\
\hline Zlat_10021635 & 1.404 & 0.026299925 & Up & TGAL3 & TGA & $\begin{array}{l}\text { Predicted: transcription factor (TF) HBP- } \\
\text { 1b(c38)-like (Oryza brachyantha) }\end{array}$ \\
\hline Zlat_10021667 & -2.160 & $1.98147 \mathrm{E}-18$ & Down & TGAL10 & TGA & $\begin{array}{l}\text { Os08g0176900, partial (Oryza sativa } \\
\text { japonica group) }\end{array}$ \\
\hline Zlat_10031473 & 3.569 & $3.70428 \mathrm{E}-11$ & Up & TGAL1 & TGA & $\begin{array}{l}\text { Hypothetical protein BRADI_2g52860v3 } \\
\text { (Brachypodium distachyon) }\end{array}$ \\
\hline Zlat_10032525 & 1.860 & $2.10235 \mathrm{E}-10$ & Up & TGAL1 & TGA & $\begin{array}{l}\text { Predicted: TF HBP- } 1 \mathrm{~b}(\mathrm{c} 1) \text {-like isoform X } 2 \\
\quad(\text { Oryza brachyantha })\end{array}$ \\
\hline Zlat_10037899 & 2.407 & 0.002668809 & Up & TGAL4 & TGA & $\begin{array}{l}\text { Predicted: TF TGA6-like (Oryza } \\
\text { brachyantha) }\end{array}$ \\
\hline Zlat_10044810 & 2.444 & $2.12905 \mathrm{E}-11$ & Up & NH5.2 & $\mathrm{TF}$ & $\begin{array}{l}\text { Hypothetical protein PAHAL_8G025500 } \\
\text { (Panicum hallii) }\end{array}$ \\
\hline \multicolumn{7}{|l|}{ Jasmonic acid (JA) } \\
\hline Zlat_10002850 & -1.121 & 0.010941287 & Down & GH3.5 & SnRK2 & $\begin{array}{l}\text { Predicted: JA-amido synthetase JAR2-like } \\
\quad \text { (Oryza brachyantha) }\end{array}$ \\
\hline Zlat_10006334 & -1.258 & 2.99678E-08 & Down & GH3.5 & SnRK2 & $\begin{array}{l}\text { Predicted: JA-amido synthetase JAR1 (Oryza } \\
\text { brachyantha) }\end{array}$ \\
\hline \multicolumn{7}{|c|}{ Indole 3-acetic acid (IAA) } \\
\hline Zlat_10000330 & 1.072 & 7.92464E-08 & Up & Os01g0856500 & SnRK2 & $\begin{array}{l}\text { Predicted: auxin (AUX) transporter-like } \\
\text { protein } 1 \text { (Oryza brachyantha) }\end{array}$ \\
\hline Zlat_10000572 & 1.070 & 0.035011956 & Up & IAA27 & PYR/PYL & $\begin{array}{l}\text { Predicted protein (Hordeum vulgare subsp. } \\
\text { vulgare) }\end{array}$ \\
\hline Zlat_10001127 & -1.261 & 0.001443811 & Down & IAA23 & $\mathrm{PP} 2 \mathrm{C}$ & $\begin{array}{l}\text { AUX-responsive protein IAA23 (Setaria } \\
\text { italica) }\end{array}$ \\
\hline Zlat_10005792 & 3.108 & $2.25714 \mathrm{E}-09$ & Up & ARF11 & $\mathrm{PP} 2 \mathrm{C}$ & $\begin{array}{l}\text { RecName: Full = AUX response factor 11; } \\
\text { AltName: Full = OsARF5 }\end{array}$ \\
\hline Zlat_10014750 & -2.970 & $2.88514 \mathrm{E}-57$ & Down & Os11g0169200 & $\mathrm{PP} 2 \mathrm{C}$ & $\begin{array}{l}\text { Predicted: putative AUX transporter-like } \\
\text { protein } 4 \text { (Oryza brachyantha) }\end{array}$ \\
\hline Zlat_10015270 & 1.248 & 0.002458758 & Up & IAA6 & $\mathrm{PP} 2 \mathrm{C}$ & $\begin{array}{l}\text { Predicted: AUX-responsive protein IAA6- } \\
\text { like isoform X3 (Oryza brachyantha) }\end{array}$ \\
\hline Zlat_10015482 & 2.632 & 7.39457E-05 & Up & LAX2 & NPR1 & $\begin{array}{l}\text { Predicted: AUX transporter-like protein } 2 \\
\quad(\text { Oryza brachyantha) }\end{array}$ \\
\hline Zlat_10015922 & 1.354 & 0.009884568 & Up & IAA7 & JAR1 & $\begin{array}{l}\text { AUX-responsive protein IAA7 } \\
\text { (Dichanthelium oligosanthes) }\end{array}$ \\
\hline Zlat_10020059 & -1.110 & $3.44535 \mathrm{E}-14$ & Down & IAA2 & JAR1 & $\begin{array}{l}\text { Hypothetical protein OsI_00719 (Oryza } \\
\text { sativa Indica Group) }\end{array}$ \\
\hline Zlat_10032090 & 1.085 & $1.12277 \mathrm{E}-06$ & Up & IAA6 & GH3 & $\begin{array}{l}\text { Predicted: AUX-responsive protein IAA6- } \\
\text { like isoform X3 (Oryza brachyantha) }\end{array}$ \\
\hline Zlat_10032626 & 1.090 & $2.93836 \mathrm{E}-15$ & Up & Os10g0147400 & CYCD3 & LAX protein (Phyllostachys edulis) \\
\hline Zlat_10033997 & -1.522 & 5.01385E-29 & Down & IAA30 & BK11 & AUX3 protein (Phyllostachys edulis) \\
\hline Zlat_10043397 & 1.079 & $1.8115 \mathrm{E}-05$ & Up & GH3.4 & B-ARR & $\begin{array}{l}\text { Hypothetical protein OsI_20501 (Oryza } \\
\text { sativa indica group) }\end{array}$ \\
\hline \multicolumn{7}{|l|}{ Gibberellin (GA) } \\
\hline Zlat_10039510 & -1.993 & 0.010678803 & Down & & B-ARR & $\begin{array}{l}\text { Hypothetical protein OsI_12756 (Oryza } \\
\text { sativa indica group) }\end{array}$ \\
\hline \multicolumn{7}{|l|}{ Cytoberberin } \\
\hline Zlat_10010976 & 1.309 & 0.002744161 & Up & RR25 & AUX1 & $\begin{array}{l}\text { Predicted: two-component response } \\
\text { regulator ORR25-like (Oryza } \\
\text { brachyantha) }\end{array}$ \\
\hline Zlat_10018529 & 1.222 & 0.006836765 & Up & RR22 & AUX1 & $\begin{array}{l}\text { Two-component response regulator ORR22 } \\
\text { (Oryza sativa japonica group) }\end{array}$ \\
\hline \multicolumn{7}{|l|}{ Brassinosteroid } \\
\hline Zlat_10018497 & 1.073 & 0.000815632 & Up & CYCD3-2 & AUX1 & $\begin{array}{l}\text { Cyclin-D3-2-like (Aegilops tauschii subsp. } \\
\text { tauschii) }\end{array}$ \\
\hline Zlat_10043001 & & 0.016310208 & Up & BKI1 & AUX1 & $\begin{array}{l}\text { Probable BRI1 kinase inhibitor } 1 \text { (Aegilops } \\
\text { tauschii subsp. tauschii) }\end{array}$ \\
\hline \multicolumn{7}{|l|}{ Abscisic acid (ABA) } \\
\hline Zlat_10005614 & -1.173 & $6.27066 \mathrm{E}-05$ & Down & PYL4 & AUX/IAA & ABA receptor 7 (Oryza sativa indica group) \\
\hline Zlat_10014121 & 1.239 & $1.83641 \mathrm{E}-07$ & Up & Os03g0268600 & AUX/IAA & $\begin{array}{l}\text { Predicted: probable protein phosphatase } 2 \mathrm{C} \\
30 \text { (Oryza brachyantha) }\end{array}$ \\
\hline Zlat_10015895 & 1.596 & 7.49921E-06 & Up & TRAB1 & AUX/IAA & $\begin{array}{l}\text { ABSCISIC ACID-INSENSITIVE 5-like } \\
\text { protein } 5 \text { (Zea mays) }\end{array}$ \\
\hline Zlat_10022265 & 4.958 & $1.22449 \mathrm{E}-05$ & Up & Os04g0167900 & AUX/IAA & $\begin{array}{l}\text { Predicted: probable protein phosphatase } 2 \mathrm{C} \\
37 \text { (Oryza brachyantha) }\end{array}$ \\
\hline Zlat_10023618 & 1.085 & $6.1928 \mathrm{E}-10$ & Up & Os05g0537400 & AUX/IAA & $\begin{array}{l}\text { Predicted: probable protein phosphatase } 2 \mathrm{C} \\
50 \text {, partial (Oryza brachyantha) }\end{array}$ \\
\hline Zlat_10029966 & 1.930 & 0.000882927 & Up & Os01g0656200 & AUX/IAA & $\begin{array}{l}\text { RecName: Full = Probable protein } \\
\text { phosphatase } 2 \mathrm{C} 8 \\
\qquad \text { (Continued on next page) }\end{array}$ \\
\hline
\end{tabular}


Table 2. (Continued from previous page)

\begin{tabular}{|c|c|c|c|c|c|c|}
\hline $\begin{array}{l}\text { Hormone category/ } \\
\text { gene ID }\end{array}$ & $\log _{2}$ fold change & $P$ value & Up/down & Gene symbol & Pathway position & Description \\
\hline Zlat_10032372 & -1.019 & 2.24929E-05 & Down & & AUX/IAA & $\begin{array}{l}\text { Hypothetical protein BRADI_2g24120v3 } \\
\text { (Brachypodium distachyon) }\end{array}$ \\
\hline Zlat_10037460 & -1.523 & 0.015159773 & Down & SAPK4 & ARF & $\begin{array}{l}\text { Os05g0433100 (Oryza sativa japonica } \\
\text { group) }\end{array}$ \\
\hline Zlat_10039544 & 1.077 & $8.18272 \mathrm{E}-09$ & Up & SAPK10 & $\mathrm{ABF}$ & $\begin{array}{l}\text { Serine/threonine-protein kinase SAPK10 } \\
\text { isoform X2 (Oryza sativa japonica group) }\end{array}$ \\
\hline Zlat_10046325 & 1.041 & 0.002060567 & Up & SAPK7 & $\mathrm{ABF}$ & $\begin{array}{l}\text { Serine/threonine-protein kinase SAPK7 } \\
\quad \text { (Oryza sativa japonica group) }\end{array}$ \\
\hline
\end{tabular}

diseases (Dean et al. 2012). Fungal infection and host defense responses are crucial events involved in plant-fungus interactions (Baker et al. 1997). Yet it is currently not fully understood how fungi alter their physiology to adapt to host environments in plant-fungus interactions. The molecular mechanism by which a plant attacks a pathogen is a complex process. $Z$. latifolia is susceptible to infection with $U$. esculenta, which stimulates the emergence and enlargement of a swollen gall under appropriate conditions (Bruce et al. 2011). Therefore, the current study provided an RNA-seq profile of TDF treatment response to infection from $U$. esculenta, which causes gall formation. Interestingly, the results showed no swollen gall formations were observable in the JB_D group while, in the JB_B group, swelling galls did form because of the phytoplasma infection (Fig. 2C). The DEG analysis of Z. latifolia gall formation indicated that several significant biological processes were altered by the $U$. esculenta infection, which provides insight into the molecular interactions between $Z$. latifolia and U. esculenta.

\section{Z. latifolia response to $U$. esculenta infection growth stage and microscopic observation.}

In previous studies, researchers have coarsely classified the growth stages of Z. latifolia (Yan et al. 2013), but their exact timing and duration (phases) were not determined. To resolve this matter, we tracked in detail the entire growth and development life cycle of $Z$. latifolia, photographing and observing each ontogenetic phase, which laid a solid foundation for studying Z. latifolia as well as for further research (Fig. 1). In the current observation of $Z$. latifolia morphological traits, we learned that the $U$. esculenta generally attached to the cell wall around it and engaged with these cells (Fig. 2). From the scanning sections, we saw massive sporophytes located in the plant cell wall, and further observations of the cell wall shows that the fungal ultrastructure is bound to it; being closely adhered to the cell wall enabled an intimate interaction between plant and fungi. U. esculenta may soften cell walls and change related genes. When $U$. esculenta interacts with plants to produce a series of metabolic reactions, plant hormone metabolism is most affected.

\section{Z. latifolia RNA-seq analysis reveal DEGs induced by $U$. esculenta.}

Many catalytically active DEGs in the infected galls were abundantly expressed throughout the gall formation process, which may be related to survival and gall cell proliferation of pathogenic fungi in host plant tissues (Kämper et al. 2006). In our study, we paid close attention to changes in the plant-type cell wall and signaling pathway, finding that $U$. esculenta can cause plants to recognize and respond to it as a host plant stimulus (Supplementary Fig. S2); accordingly, this would suggest that $U$. esculenta could play a significant role in protecting plants from other fungal infections ( $\mathrm{Li}$ et al. 2007). Secondary metabolites, such as lignin biosynthesis, via the phenylpropanoid biosynthesis pathway, can promote plant resistance to pathogens (Rubery and Fosket 1969). We found that most of the MYB TF was up-regulated in the JB_B group (Fig. 6) and that MYB could regulate the pathways of lignin biosynthesis and phenylpropanoid biosynthesis. This may provide opportunities for the emergence and expansion of cell-wall resistance in multiple plant tissues.

TFs play a central role in plant defense (Singh et al. 2002), which involves a number of transcriptional regulations triggered by endophytic fungi in a host to produce a series of responses to attacking endophytic fungi. TF is a protein that, by binding to specific DNA sequences, regulates genes in a coordinated manner, thereby directing cell division, development, and death. In our study, most TFs were significantly up- or down-regulated (Supplementary Table S2C) in response to $U$. esculenta infection. Transcriptome and qPCR expression analyses of selected DEGs indicated that these TFs could regulate transcriptional changes in response to $U$. esculenta infection. Further research should focus on molecular mechanisms of these TFs to better understand the different regulation mechanisms of IAA-related DEGs conferring a coordinated plant response to $U$. esculenta.

\section{Expression levels of DEGs involved in plant hormone signal transduction changed significantly after U. esculenta infection.}

Plant hormones play a role in a complex network that regulates plant growth, gall formation, and plant responses to environmental signals (Denancé et al. 2013; Jaillais and Chory 2010; Pieterse et al. 2009). The role of plant-derived hormones in phytoplasma infection resistance has been widely reviewed (De Vleesschauwer et al. 2010; Roberts et al. 2011). Meanwhile, pathogens have evolved complex mechanisms to regulate hormone metabolism and signal transduction in their hosts, thereby promoting their ability to overcome plant defense mechanisms (Dodds and Rathjen 2010). Some hormones, such as IAA and ABA, figure prominently in regulating plant growth and responding to abiotic stresses, but they have recently been shown to play an important role in plant-fungal interactions also (Fu and Wang 2011; Ton et al. 2009). Similarly, in this study, we also observed that $U$. esculenta infection affected gene expression levels related to IAA, CTK, and GA signaling and metabolism. Therefore, during the $U$. esculenta infection period, all plant hormone pathways were linked to each other in a large and complex network, and Z. latifolia appears to activate all regulators of IAA, CTK, GA, and ABA in response to endogenous symbiosis with the fungus. Future research on this interaction should focus on detailed comparisons of JB_D versus JB_Bmediated endophytic fungal infection of plants. The majority of genes involved in plant hormone signal transduction of IAA, CTK, and GA were down-regulated and ABA, SA, and ETH were up-regulated at JB_D versus JB_B. We speculate that $U$. esculenta targets the host plant hormone system during its main invasion phase and that these downregulated DEGs may be a host protective response with some stimulants secreted. Those DEGs related to IAA were down-regulated, which may be linked to the higher content of IAA present when broom symptoms are present in pathogenic infections (Zafari et al. 2012). 
In the current study, most genes involved in IAA metabolism and signal pathway were down-regulated (e.g., SAUR39, PLC2) in JB_D versus JB_B plants of Z. latifolia. Moreover, expression of the SAUR39 gene was rapidly augmented under the action of an AUX-responsive protein, and SAUR39 is thought to play a significant role in the local regulation of AUX polar transport in plant responses against $U$. esculenta infection (Kant et al. 2009). The ARF genes are central components of plant AUX metabolism and signal transduction. The IAA content was greatly increased in the JB_B group (Figs. 3 and 8; Supplementary Table S6), which exhibited the highest expression change among the IAArelated genes. Therefore, the gene for SAUR39 and ARF TFs could be key components for regulating how plants respond to $U$. esculenta infection.

From the KEGG enrichment pathways, we found that "phytohormone signal transduction pathway' and 'phenylpropanoid biosynthesis pathway' had the highest concentrations of KEGG pathways (Fig. 5). In the process of fungal infection, plants usually synthesize flavonoids and lignin by using phenylpropanoid compounds (Dixon et al. 2002). In many plant species, including Arabidopsis and Oryza sativa, upregulation of $A U X / I A A$ and $A R F$ genes after gall swelling occurs downstream of IAA signal transduction (Bridge et al. 2012; Song et al. 2009). Moreover, previous work has shown that symptomatic Arabidopsis plants infected by bacteria had detectable increases in their IAA content (Dolzblasz et al. 2018); similarly, the IAA content was substantially increased in Z. latifolia in the JB_B group (Fig. 3A). The $A R F$ gene is a crucial component of an alternative pathway leading to IAA metabolism and signaling transduction (Bargmann and Estelle 2014). AUX positively controls the plants' gall expanse, growth, and development, whereas IAA pathways can commonly regulate endophytic fungi quite effectively (Khan et al. 2012; Mehmood et al. 2018).
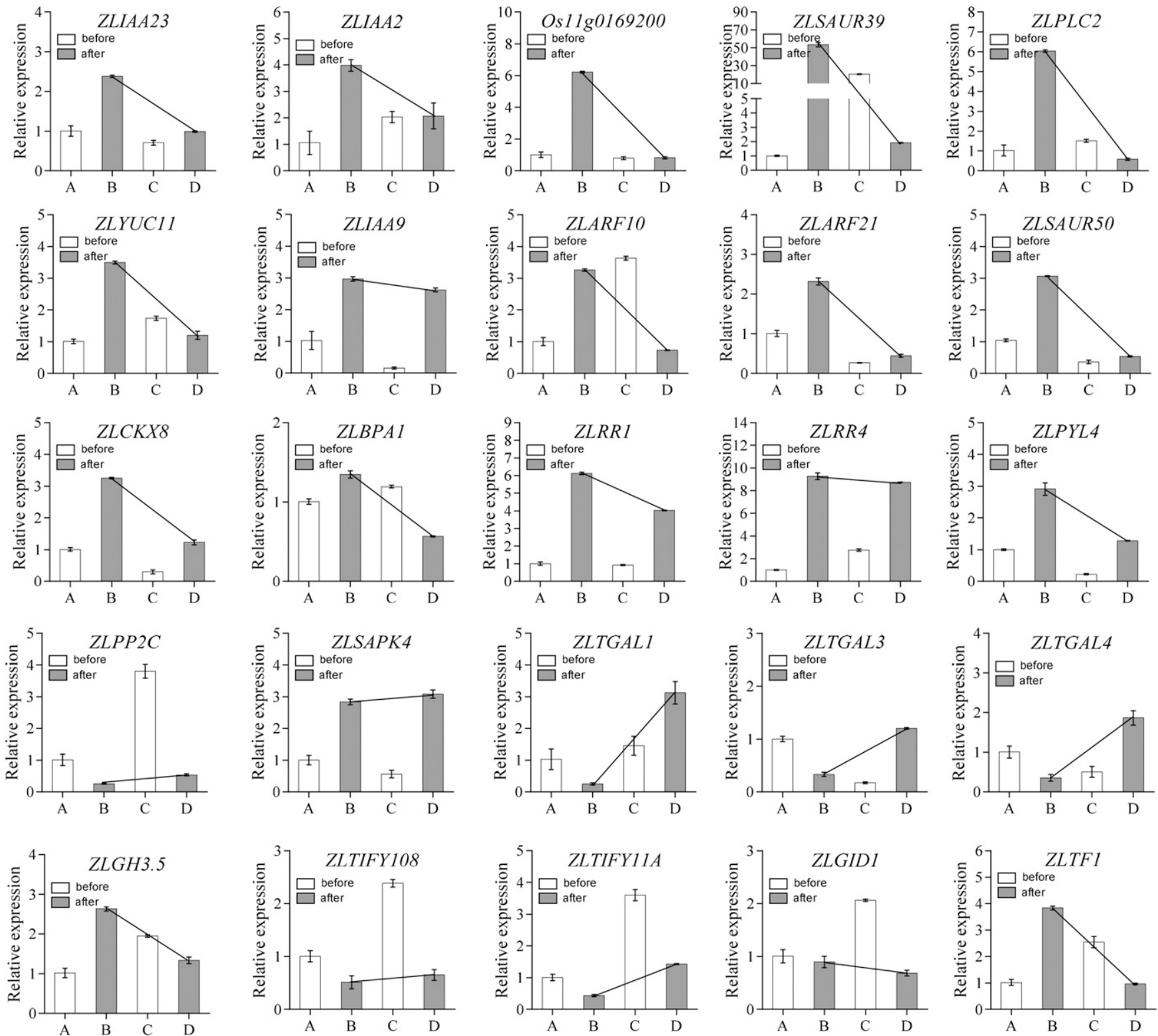

Fig. 8. Expression analysis of hormone-related genes in different groups. The experiment included three biological replicates and used SPSS for the analysis. We chose the following number of hormone-related differentially expressed genes: 10 related to indole 3-acetic acid, four to cytoberberin, three to abscisic acid, three to salicylic acid, three to jasmonic acid, and two to gibberellin. In all panels, the $x$ axis represents the four sample groups: A (JB_A), B (JB_B), C (JB_C), and D (JB_D). 
Most of the DEGs related to both IAA and CTK pathways had down-regulated expression in JB_D versus JB_B, resulting in greater JA and SA contents at the initial stage of $U$. esculenta infection in the JB_B group. It seems the combination of DEGs associated with IAA and CTK hormones has been changed to either foster a synergistic effect against $U$. esculenta infection or is simply a part of the evolved symbiotic relationship between $Z$. latifolia and $U$. esculenta. The hormone ABA

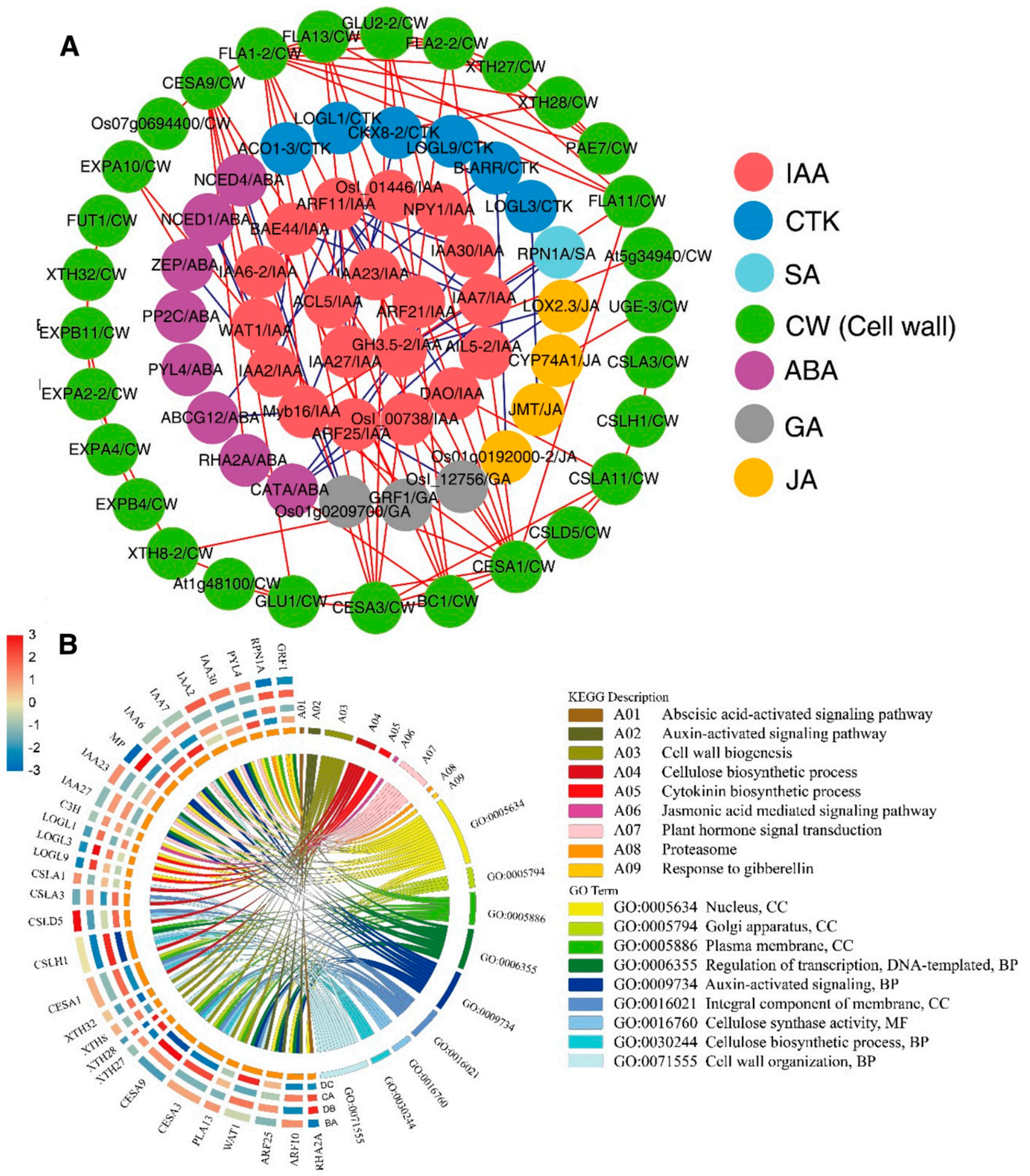

Fig. 9. The interaction network was constructed by plant hormone and cell wall-relative genes in Zizania latifolia. A, We used interaction network maps to draw the interaction networks among six key plant hormone-related genes (indole 3-acetic acid [IAA], cytoberberin [CTK], salicylic acid [SA], gibberellin [GA], jasmonic acid [JA], abscisic acid [ABA]) and cell-wall wall loosening-related genes (IAA in red, CTK in blue, SA in light blue, cell wall in green, ABA in purple, GA in gray, JA in yellow), finding that cell wall-loosening factors clustered around the hormones. Auxin is the center and other hormones regulated the expansion of gall formations in Z. latifolia. B, The major genes were analyzed by gene ontology and Kyoto Encyclopedia of Genes and Genomes. The heatmap in each group is represented in the left half. 
integrates diverse stress signals to regulate downstream stress response of plants (Hauser et al. 2011; Tuteja 2007). In our study, the DEGs involved in the ABA signal transduction pathway (including $A B F, P P 2 C, P Y L / P Y L$, and $S N R K 2$ ) were significantly down-regulated in the JB_D versus JB_B comparison (Fig. 7; Supplementary Table S6). $P P 2 C$ is a feedback regulation gene in ABA signaling and transcriptionally regulates expression of the ABA-responsive gene (Wang et al. 2019). Therefore, downregulation of the PP2C gene indicated that ABA signaling might have been reduced in the plant after its infection with $U$. esculenta. In addition, plant immune signals become activated to inhibit diseases caused by the response effects of ABA signaling (Kim 2012). This result may indicate activation of ABA signaling during gall formation of $U$. esculenta postinfection, but, when the JB_B immune system is recognized, the ABA content undergoes a relative decline.

\section{$U$. esculenta response to the host is to soften cell walls and further produce large amounts of hormones to promote gall swelling.}

A hormone regulation model with plant hormone signal transaction and cell wall-loosening genes functional during U. esculenta infection in Z. latifolia is depicted in Figures 9 and 10. We devised a working model (Fig. 10) in which IAA plays a significant role in regulating $U$. esculenta infection in $Z$. latifolia plants. On the one hand, $U$. esculenta infection

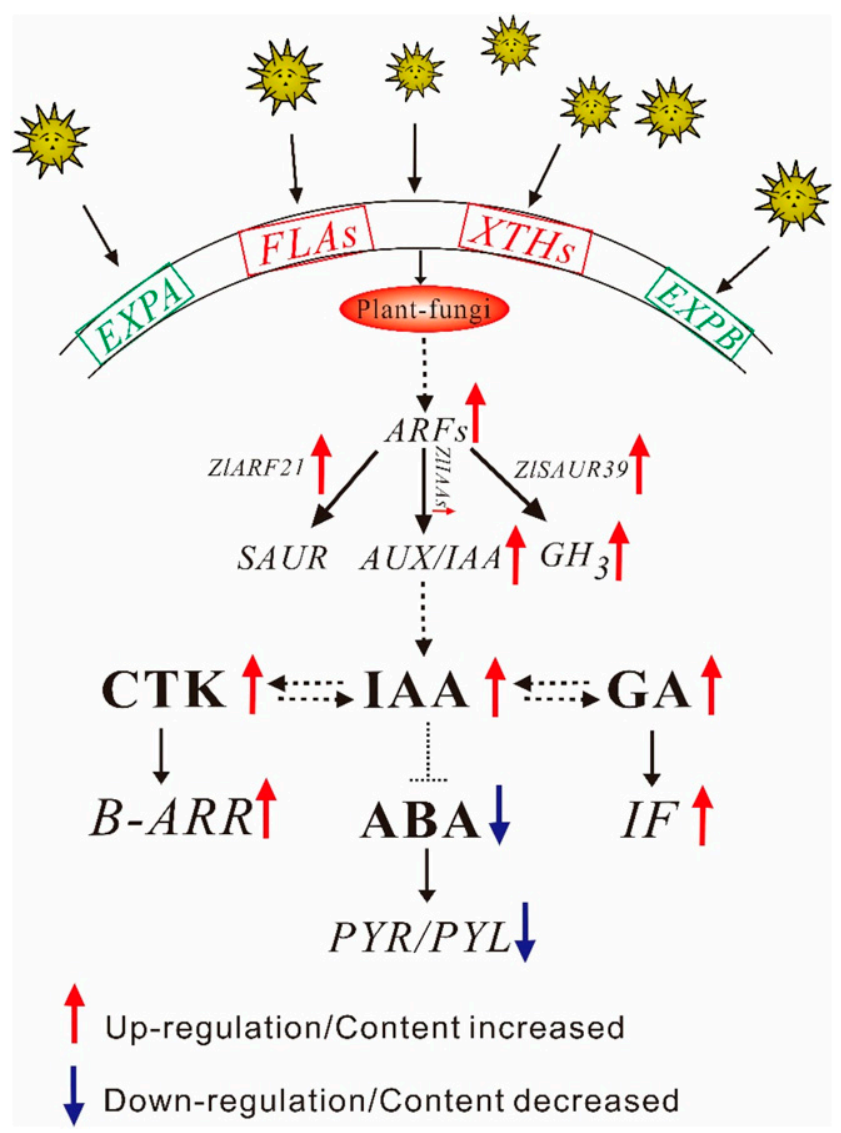

Fig. 10. Proposed hormone-cell wall loosening model of Zizania latifolia plants infected with Ustilago esculenta. The fungus $U$. esculenta infects the cells via the cell wall, softening it, eliciting changes in the activity of cell wall-loosening-related genes (ZlEXPA, ZlFLAs, ZlXTHs, and ZlEXPB). Dashed lines indicate that further research is needed. Italics represent genes, the red arrows indicate upregulation or increased content, and the blue arrows indicate downregulation or decreased content. reduces the IAA level and upregulates $A U X 1, A U X / I A A$, and $A R F$ gene family expression. CTK synthesis is negatively regulated by IAA, and this process depends on the inhibition of B-ARR (Müller and Leyser 2011). One of the dominating responses was enhanced CTK signaling, which may be due to B-ARR-related gene upregulation. Elevated CTK-related gene expression levels and content generate a range of reactions, including cell division, shoot initiation, and gall enlargement. The other response to $P Y R / P Y L$ downregulation is decreased ABA synthesis, followed by upregulation of the $X T H \mathrm{~s}, F L A \mathrm{~s}, E X P A$, and $E X P B$ required for the cell wallloosening factor (Figs. 9 and 10). On the other hand, U. esculenta infection stimulates IAA transport and synthesis, which diminishes GA catabolism. However, an increased GA3 content in $Z$. latifolia, possibly by downregulation of $\mathrm{TF}$ genes, was also observed in our study, and ABA synthesis was accordingly reduced in response. Additionally, U. esculenta infection leads to the upregulation of $\mathrm{ABF}$, resulting in the rapid reduction of ABA transduction via SnRK2 activity, which are negative signals for the formation of swelling galls in Z. latifolia (Fig. 10). It seems reasonable, then, to infer that host transcriptome hormonal adjustments and cell-wall loosening in this model constitute a key fine-tuning process for the coexistence of $U$. esculenta and $Z$. latifolia. The former can loosen the latter's cell wall, thereby gaining access to the interior of host cells, which stimulates a series of hormone responses in symbiosis with $Z$. latifolia, utilizing in novo activation of swelling gall formation.

\section{MATERIALS AND METHODS}

Plant materials and growth conditions.

The variety used in the experiments was the single-season Zizania latifolia cv. Dabieshan No.1, purchased from Yuexi County (Yuexi County Longjing Ecological Agriculture Development Co., Ltd., Anhui, China). Photographs (front view, cross-section, and vertical section) were taken of each plant growth stage to confirm the sampling and treatment periods for experimentation (Fig. 1). Samples, which included the swelling stem, were immediately frozen, using liquid nitrogen flashfreezing, and were stored at $-80^{\circ} \mathrm{C}$ for later use in the relative gene expression research and experiments.

For transcriptome assembly, to test and quantify the effect of swelling gall formation, TDF was used because it prevents and inhibits fungal activity in Z. latifolia. The exogenous application of TDF ( $80 \mathrm{mg}$ per liter) was carried out twice, before and after gall formation, and the spray was applied at intervals of 5 days. Given the known development of Z. latifolia, these plants were treated twice, 148 (on September 15, 2018) and 159 (on September 26, 2018) days after transplanting. For subsequent experiments, gall samples were collected at 5 days before and 5 days after gall formation, with minimum gall diameters of approximately $5 \mathrm{~cm}$. Some swelling galls were harvested at each timepoint, for which their sampling was done in triplicate, using freeze tube storage. All samples were placed in cryopreservation tubes and were immediately stored in liquid nitrogen in a refrigerator at $-80^{\circ} \mathrm{C}$ for RNA extraction and several index determinations. Using a $2 \times 2$ factorial design, we harvested four groups of samples: $\mathrm{CK}$ (control: no treatment) versus TDF-treated $Z$. latifolia plants, sampled either before or after the swollen gall formation occurred in them. The four groups were called JB-A (CK, before swelling gall formation of Z. latifolia), JB-B (CK, after swelling gall formation of Z. latifolia), JB-C (TDF treatment, before swelling gall formation of Z. latifolia), JB-D (TDF treatment, after swelling gall formation of $Z$. latifolia). All experiments were repeated in triplicate. 
Microscopic observations before and after gall formation in the CK and TDF plants.

To study the interaction relationships of the above four experimental groups, their respective swollen gall samples were stained using aniline blue (CAS number 28631-66-5; Solarbio) according to an improved method (Faoro et al. 2008). Fresh stem tip samples of $Z$. latifolia tissue were sliced and fixed with a fluid containing the Carnot fixative (glacial acetic acid and alcohol, volume ratio of 1:3) and were put into a 2-ml centrifuge tube, were stored in refrigerator at $4^{\circ} \mathrm{C}$ for $24 \mathrm{~h}$. To it was added the right amount of $10 \% \mathrm{KOH}$ solution, after which it was subjected to a $85^{\circ} \mathrm{C}$ high-temperature treatment lasting $1 \mathrm{~h}$; it was washed several times during the $\mathrm{KOH}$ solution and, after processing, was used for subsequent dyeing observation. The slices were transferred into a Petri dish containing aniline blue dye and were stained on a horizontal shaker for 5 to $10 \mathrm{~min}$. After decolorization with $75 \%$ alcohol, the slices were observed and photographed under a fluorescence microscope (Olympus SZX10). Then, paraffin sections were made and stained with aniline blue for observation under an optical microscope (Nikon Eclipse E100).

To investigate the effect of exogenous TDF on gall swelling and formation at the biological level, the ultrastructure of the gall tip cell was observed using electron microscopy with the following method (Chen et al. 2019). Specifically, the slices were observed under a transmission electron microscope, set to an accelerating voltage of $80 \mathrm{kv}$ (H-7650, Hitachi). In parallel, a scanning electron microscope (Hitachi S-4800) was used to observe the $U$. esculenta spores (Waikhom et al. 2013).

\section{Determination of the plant endogenous hormone contents.}

The swollen galls were analyzed before and after gall formation in CK and TDF. The extraction, purification, and determination of endogenous IAA, Z+ZR, $\mathrm{GA}_{3}$, and $\mathrm{ABA}$ by UHPLC followed methodologies described before ( $\mathrm{Li}$ et al. 2019; Yuan et al. 2008). For these determinations, we used the mixed labeling method to distinguish the respective content of these five hormones in the plants.

Hormone contents were measured by UHPLC, four samples were collected, and the extraction, purification, and measurement protocols were already described by Li et al. (2019). To analyze the hormone contents, a Thermo Fisher Scientific UHPLC UltiMate 3000 was used, equipped with a vacuum degasser, quantitative pump, automatic sampler, thermostatic column chamber, and fluorescence detector. A BETASIL C18 column (Thermo Fisher Scientific; $4.6 \times 250 \mathrm{~mm}, 5 \mathrm{~mm}$ ) and fluorescence detection wavelength of Eml 1/4 $254 \mathrm{~nm}$ were both use. Each sample $(10 \mathrm{ml})$ was injected automatically into the UHPLC system, at a flow rate of $1 \mathrm{ml} \mathrm{min}^{-1}$. Quantifications were made by comparing the peak area of a detected compound with the known dose of that hormone.

\section{cDNA library construction and in novo assembly.}

As for the microscopic observations and analyses described above, RNA-seq analysis was also performed before and after the gall formation of CK and TDF plants, from which a total of 12 gall-tip samples were taken. To simplify their description and results reporting, we followed the same protocol used for naming the early four groups in naming their three replicates, i.e., JB_A, JB_B, JB_C, and JB_D, respectively. Total RNA was extracted from 12 gall samples before and after gall formation in CK and TDF by using the mir-Vana miRNA isolation kit (mirVana miRNA ISOlation kit; Ambion) following manufacturer protocol. The group-specific libraries were constructed with the TruSeq Stranded mRNA LTSample prep kit (Illumina), according to manufacturer instructions. These cDNA libraries were then sequenced on the Illumina sequencing platform (Illumina HiSeq 2500) to generate 125 $\mathrm{bp} / 150$-bp paired-end reads. Then, transcriptome sequencing and raw reads were processed by Trimmomatic (Bolger et al. 2014). To obtain clear reads, those reads containing ploy-N and any low-quality reads were first removed. The ensuing clean reads were then mapped onto the reference genome (in the RiceRelatives genomic database), using the 'HISAT2' alignment software program (Kim et al. 2015). A small proportion of $U$. esculenta was filtered out directly by comparisons with the Z. latifolia genome.

\section{Analysis of DEGs, cluster analysis, and GO and KEGG enrichments.}

The FPKM (Roberts et al. 2011) value of all genes was calculated by the Cufflinks tool (Trapnell et al. 2010), and the read counts for all genes were obtained using the 'htseq-count' tool (Anders et al. 2015). For transcriptional-level quantifications, the FPKM (Kim et al. 2015) and read counts(protein coding) values for all transcripts were calculated by Bowtie2 (Langmead and Salzberg 2012) and eXpress (Roberts and Pachter 2013). The DEGs were identified using the DESeq package in R (Anders and Huber 2013), which relied on its function estimation size factor and nbinom test; a $P$ value $<0.05$ and absolute fold change $>2$ or $<0.5$ were the threshold criteria for designating a gene having significant differential expression among the group pairings. Hierarchical clustering analysis of DEGs was analyzed to further explore patterns of gene expression. Based on the hypergeometric distribution, GO enrichment and KEGG (Kanehisa et al. 2008) pathway enrichment analysis of identified DEGs were respectively implemented in the R program (version: 3.6.1). The read portions were reassembled by StringTie (Pertea et al. 2015), after which the reference genome and known annotated genes were aligned, using the Cuffcompare software program, for both gene structure extensions and new transcript identifications.

\section{Functional annotation and KEGG enrichment pathway analysis.}

Identified DEGs were characterized by GO and KEGG enrichment (KEGG database) to characterize biological functions and significantly enriched metabolic pathways or signal transduction pathways. Based on Wallenius' noncentral hypergeometric distribution (Young et al. 2010), DEGs in our study were submitted to GO enrichment analysis, using the 'GO-seq' $\mathrm{R}$ package, for their enrichment analysis. The statistical evidence for significant enrichment of DEGs in the KEGG pathway was detected by KOBAS 3.0 (Mao et al. 2005) online. Finally, the MapMan graphing tool was used to depict an overview of the metabolic and regulatory pathways.

\section{qRT-PCR analysis.}

To explore the expression patterns of genes participating in gall formation, 10 IAA, four ABA, three CTK, three SA, three JA, two GA-related genes were analyzed in CK and TDF treatment, using real-time qPCR and improved methodology (Chen et al. 2018). Total RNA samples were isolated from different stages with the RNA-prep Pure plant kit (Tiangen). The DNase-treated RNA was extracted and reverse transcribed into cDNA by the Prime Script RT reagent kit (TaKaRa), after which real-time qPCR was implemented with the TB Green Premix Ex Taq II (TaKaRa). The relative expression level of the housekeeping Actin gene served as an internal reference for standardizing the data. Three biological replicates were performed per sample, and the relative expression levels were calculated as $2^{-\Delta \Delta \mathrm{Ct}}$ (Livak and Schmittgen 2001). The realtime qPCR primer sequences used for this can be found in Supplementary Table S5. These results are displayed as the 
mean \pm standard deviation (biological replicates, $n=3$ ). The relative expression data were statistically analyzed using SPSS software. Every sample consisted of three biological replicates.

\section{Statistical analysis.}

Our experimental work used a completely randomized block design. The data were analyzed in SPSS software, by applying analysis of variance and using Duncan's multiple range test. $P<$ 0.05 was considered significant.

\section{ACKNOWLEDGMENTS}

Thanks to the biotechnology center at the Horticulture College of Anhui Agricultural University for providing experimental support.

\section{AUTHOR-RECOMMENDED INTERNET RESOURCES}

KEGG database: http://www.genome.jp/kegg

KOBAS 3.0 analysis tool: http://kobas.cbi.pku.edu.cn

MapMan graphing tool: https://mapman.gabipd.org

RiceRelatives genomic database: http://ibi.zju.edu.cn/ricerelativesgd

\section{LITERATURE CITED}

Ahmad, P., and Prasad, M. N. V. 2012. Abiotic Stress Responses in Plants. Springer, New York.

Anders, S., and Huber, W. 2013. Differential expression of RNA-seq data at the gene level-The DESeq package. European Molecular Biology Laboratory, Heidelberg, Germany.

Anders, S., Pyl, P. T., and Huber, W. 2015. HTSeq-A Python framework to work with high-throughput sequencing data. Bioinformatics 31 : 166-169.

Baker, B., Zambryski, P., Staskawicz, B., and Dinesh-Kumar, S. P. 1997. Signaling in plant-microbe interactions. Science 276:726-733.

Bargmann, B. O., and Estelle, M. 2014. Auxin perception: In the IAA of the beholder. Physiol. Plant. 151:52-61.

Bhargava, S., and Sawant, K. 2013. Drought stress adaptation: Metabolic adjustment and regulation of gene expression. Plant Breed. 132:21-32.

Bolger, A. M., Lohse, M., and Usadel, B. 2014. Trimmomatic: A flexible trimmer for Illumina sequence data. Bioinformatics 30:2114-2120.

Bridge, L. J., Mirams, G. R., Kieffer, M. L., King, J. R., and Kepinski, S. 2012. Distinguishing possible mechanisms for auxin-mediated developmental control in Arabidopsis: Models with two Aux/IAA and ARF proteins, and two target gene-sets. Math. Biosci. 235:32-44.

Bruce, S. A., Saville, B. J., and Neil Emery, R. J. 2011. Ustilago maydis produces cytokinins and abscisic acid for potential regulation of tumor formation in maize. J. Plant Growth Regul. 30:51-63.

Chaves, M. M., Maroco, J. P., and Pereira, J. S. 2003. Understanding plant responses to drought-From genes to the whole plant. Funct. Plant Biol. 30:239-264.

Chen, G., Ye, X., Zhang, S., Zhu, S., Yuan, L., Hou, J., and Wang, C. 2018. Comparative transcriptome analysis between fertile and CMS flower buds in Wucai (Brassica campestris L.). BMC Genomics 19:908.

Chen, G., Zeng, F., Wang, J., Ye, X., Zhu, S., Yuan, L., Hou, J., and Wang, C. 2019. Transgenic Wucai (Brassica campestris L.) produced via Agrobacterium-mediated anther transformation in planta. Plant Cell Rep. 38:577-586.

Chung, K. R., and Tzeng, D. D. 2004. Biosynthesis of indole-3-acetic acid by the gall-inducing fungus Ustilago esculenta. J. Biol. Sci. 4: 744-750.

De Vleesschauwer, D., Yang, Y., Cruz, C. V., and Höfte, M. 2010. Abscisic acid-induced resistance against the brown spot pathogen Cochliobolus miyabeanus in rice involves MAP kinase-mediated repression of ethylene signaling. Plant Physiol. 152:2036-2052.

Davies, W. J., Kudoyarova, G., and Hartung, W. 2005. Long-distance ABA signaling and its relation to other signaling pathways in the detection of soil drying and the mediation of the plant's response to drought. J. Plant Growth Regul. 24:285-295.

Dean, R., Van Kan, J. A., Pretorius, Z. A., Hammond-Kosack, K. E., Di Pietro, A., Spanu, P. D., Rudd, J. J., Dickman, M., Kahmann, R., Ellis, J., and Foster, G. D. 2012. The top 10 fungal pathogens in molecular plant pathology. Mol. Plant Pathol. 13:414-430.

Denancé, N., Sánchez-Vallet, A., Goffner, D., and Molina, A. 2013. Disease resistance or growth: The role of plant hormones in balancing immune responses and fitness costs. Front. Plant Sci. 4:155.
Dixon, R. A., Achnine, L., Kota, P., Liu, C. J., Reddy, M. S., and Wang, L. 2002. The phenylpropanoid pathway and plant defence-A genomics perspective. Mol. Plant Pathol. 3:371-390.

Dodds, P. N., and Rathjen, J. P. 2010. Plant immunity: Towards an integrated view of plant-pathogen interactions. Nat. Rev. Genet. 11: 539-548.

Doehlemann, G., Wahl, R., Horst, R. J., Voll, L. M., Usadel, B., Poree, F., Stitt, M., Pons-Kühnemann, J., Sonnewald, U., Kahmann, R., and Kämper, J. 2008. Reprogramming a maize plant: Transcriptional and metabolic changes induced by the fungal biotroph Ustilago maydis. Plant J. 56:181-195.

Dolzblasz, A., Banasiak, A., and Vereecke, D. 2018. Neovascularization during leafy gall formation on Arabidopsis thaliana upon Rhodococcus fascians infection. Planta 247:215-228.

Doonan, J. H., and Sablowski, R. 2010. Walls around tumours-Why plants do not develop cancer. Nat. Rev. Cancer 10:794-802.

Faoro, F., Maffi, D., Cantu, D., and Iriti, M. 2008. Chemical-induced resistance against powdery mildew in barley: The effects of chitosan and benzothiadiazole. BioControl 53:387-401.

Fu, J., and Wang, S. 2011. Insights into auxin signaling in plant-pathogen interactions. Front. Plant Sci. 2:74.

Gelvin, S. B. 2003. Agrobacterium-mediated plant transformation: The biology behind the "gene-jockeying" tool. Microbiol. Mol. Biol. Rev. 67: 16-37.

Guo, H. B., Li, S. M., Peng, J., and Ke, W. D. 2007. Zizania latifolia Turcz. cultivated in China. Genet. Resour. Crop Evol. 54:1211-1217.

Guo, L., Qiu, J., Han, Z., Ye, Z., Chen, C., Liu, C., Xin, X., Ye, C. Y., Wang, Y. Y., Xie, H., Wang, Y., Bao, J., Tang, S., Xu, J., Gui, Y., Fu, F., Wang, W., Zhang, X., Zhu, Q., Guang, X., Wang, C., Cui, H., Cai, D., Ge, S. Tuskan, G. A., Yang, X., Qian, Q., He, S. Y., Wang, J., Zhou, X. P., and Fan, L. 2015. A host plant genome (Zizania latifolia) after a century-long endophyte infection. Plant J. 83:600-609.

Hahn, M., and Mendgen, K. 2001. Signal and nutrient exchange at biotrophic plant-fungus interfaces. Curr. Opin. Plant Biol. 4:322-327.

Han, S., Zhang, H., Qin, L., and Zhai, C. 2013. Effects of dietary carbohydrate replaced with wild rice (Zizania latifolia (Griseb) Turcz) on insulin resistance in rats fed with a high-fat/cholesterol diet. Nutrients 5:552-564.

Hauser, F., Waadt, R., and Schroeder, J. I. 2011. Evolution of abscisic acid synthesis and signaling mechanisms. Curr. Biol. 21:R346-R355.

Hirsch, A. M., Fang, Y., Asad, S., and Kapulnik, Y. 1997. The role of phytohormones in plant-microbe symbioses. Plant Soil 194:171-184.

Horst, R. J., Engelsdorf, T., Sonnewald, U., and Voll, L. M. 2008. Infection of maize leaves with Ustilago maydis prevents establishment of $\mathrm{C} 4$ photosynthesis. J. Plant Physiol. 165:19-28.

Hossain, M. Z., Ishiga, Y., Yamanaka, N., Ogiso-Tanaka, E., and Yamaoka, Y. 2018. Soybean leaves transcriptomic data dissects the phenylpropanoid pathway genes as a defence response against Phakopsora pachyrhizi. Plant Physiol. Biochem. 132:424-433.

Jaillais, Y., and Chory, J. 2010. Unraveling the paradoxes of plant hormone signaling integration. Nat. Struct. Mol. Biol. 17:642-645.

Kämper, J., Kahmann, R., Bölker, M., Ma, L. J., Brefort, T., Saville, B. J., Banuett, F., Kronstad, J. W., Gold, S. E., Müller, O., Perlin, M. H., Wösten, H. A., de Vries, R., Ruiz-Herrera, J., Reynaga-Peña, C. G., Snetselaar, K., McCann, M., Pérez-Martín, J., Feldbrügge, M., Basse, C. W., Steinberg, G., Ibeas, J. I., Holloman, W., Guzman, P., Farman, M., Stajich, J. E., Sentandreu, R., González-Prieto, J. M., Kennell, J. C., Molina, L., Schirawski, J., Mendoza-Mendoza, A., Greilinger, D., Münch, K., Rössel, N., Scherer, M., Vranes, M., Ladendorf, O., Vincon, V., Fuchs, U., Sandrock, B., Meng, S., Ho, E. C., Cahill, M. J., Boyce, K. J., Klose, J., Klosterman, S. J., Deelstra, H. J., Ortiz-Castellanos, L., Li, W., Sanchez-Alonso, P., Schreier, P. H., Häuser-Hahn, I., Vaupel, M., Koopmann, E., Friedrich, G., Voss, H., Schlüter, T., Margolis, J., Platt, D., Swimmer, C., Gnirke, A., Chen, F., Vysotskaia, V., Mannhaupt, G., Güldener, U., Münsterkötter, M., Haase, D., Oesterheld, M., Mewes, H. W., Mauceli, E. W., DeCaprio, D., Wade, C. M., Butler, J., Young, S., Jaffe, D. B., Calvo, S., Nusbaum, C., Galagan, J., and Birren, B. W. 2006. Insights from the genome of the biotrophic fungal plant pathogen Ustilago maydis. Nature 444:97-101.

Kanehisa, M., Araki, M., Goto, S., Hattori, M., Hirakawa, M., Itoh, M. Katayama, T., Kawashima, S., Okuda, S., Tokimatsu, T., and Yamanishi, Y. 2008. KEGG for linking genomes to life and the environment. Nucleic Acids Res. 36:D480-D484.

Kant, S., Bi, Y. M., Zhu, T., and Rothstein, S. J. 2009. SAUR39, a small auxin-up RNA gene, acts as a negative regulator of auxin synthesis and transport in rice. Plant Physiol. 151:691-701.

Khan, A. L., Shinwari, Z. K., Kim, Y. H., Waqas, M., Hamayun, M., Kamran, M., and Lee, I. J. 2012. Role of endophyte chaetomium 
globosum Lk4 in GROWTH of Capsicum апnиum by producion of gibberellins and indole acetic acid. Pak. J. Bot. 44:1601-1607.

Kim, T. H. 2012. Plant stress surveillance monitored by ABA and disease signaling interactions. Mol. Cells 33:1-7.

Kim, D., Langmead, B., and Salzberg, S. L. 2015. HISAT: A fast spliced aligner with low memory requirements. Nat. Methods 12:357-360.

Langmead, B., and Salzberg, S. L. 2012. Fast gapped-read alignment with Bowtie 2. Nat. Methods 9:357-359.

Li, J., Guan, Y., Yuan, L., Hou, J., Wang, C., Liu, F., Yang, Y., Lu, Z., Chen, G., and Zhu, S. 2019. Effects of exogenous IAA in regulating photosynthetic capacity, carbohydrate metabolism and yield of Zizania latifolia. Sci. Hortic. (Amsterdam) 253:276-285.

$\mathrm{Li}, \mathrm{S}$. 2016. Cloning and expression analysis of resistan genes $\mathrm{Zl-RPM1}$ and $Z l-A D R$ in Zizinia latifolia. Research Square. Published online. doi: $10.21203 / \mathrm{rs} .2 .11144 / \mathrm{v} 1$

Li, Y., Beisson, F., Koo, A. J. K., Molina, I., Pollard, M., and Ohlrogge, J. 2007. Identification of acyltransferases required for cutin biosynthesis and production of cutin with suberin-like monomers. Proc. Natl. Acad. Sci. U.S.A. 104:18339-18344.

Livak, K. J., and Schmittgen, T. D. 2001. Analysis of relative gene expression data using real-time quantitative PCR and the $2^{-\Delta \Delta \mathrm{C}(\mathrm{T})}$ method. Methods 25:402-408.

Mao, X., Cai, T., Olyarchuk, J. G., and Wei, L. 2005. Automated genome annotation and pathway identification using the KEGG Orthology (KO) as a controlled vocabulary. Bioinformatics 21:3787-3793.

Mehmood, A., Hussain, A., Irshad, M., Khan, N., Hamayun, M., Ismail, Afridi, S. G., and Lee, I.-J. 2018. IAA and flavonoids modulates the association between maize roots and phytostimulant endophytic Aspergillus fumigatus greenish. J. Plant Interact. 13:532-542.

Mizoi, J., Shinozaki, K., and Yamaguchi-Shinozaki, K. 2012. AP2/ERF family transcription factors in plant abiotic stress responses. Biochim. Biophys. Acta 1819:86-96.

Moghadasian, M. H., Kaur, R., Kostal, K., Joshi, A. A., Molaei, M., Le, K., Fischer, G., Bonomini, F., Favero, G., Rezzani, R., Gregorchuk, B. S. J., Leung-Shing, V., Wuzinski, M., Seo, A. I., and Bay, D. C. 2019. Antiatherosclerotic properties of wild rice in low-density lipoprotein receptor knockout mice: The gut microbiome, cytokines, and metabolomics study. Nutrients 11:2894.

Morrison, E. N., Emery, R. J., and Saville, B. J. 2015. Phytohormone involvement in the Ustilago maydis- Zea mays pathosystem: Relationships between abscisic acid and cytokinin levels and strain virulence in infected cob tissue. PLoS One 10:e0130945.

Müller, D., and Leyser, O. 2011. Auxin, cytokinin and the control of shoot branching. Ann. Bot. 107:1203-1212.

Pertea, M., Pertea, G. M., Antonescu, C. M., Chang, T. C., Mendell, J. T., and Salzberg, S. L. 2015. StringTie enables improved reconstruction of a transcriptome from RNA-seq reads. Nat. Biotechnol. 33:290-295.

Pieterse, C. M., Leon-Reyes, A., Van der Ent, S., and Van Wees, S. C. 2009. Networking by small-molecule hormones in plant immunity. Nat. Chem. Biol. 5:308-316.

Qingsen, Z. 2006. Some biological characteristics of Ustilago esculenta in different Zizania latifolia cultivars. Jiangsu J. Agr. Sci. 22:71-75.

Quan, Z., Pan, L., Ke, W., Liu, Y., and Ding, Y. 2009. Sixteen polymorphic microsatellite markers from Zizania latifolia Turcz. (Poaceae). Mol. Ecol. Resour. 9:887-889.

Rabe, F., Ajami-Rashidi, Z., Doehlemann, G., Kahmann, R., and Djamei, A. 2013. Degradation of the plant defence hormone salicylic acid by the biotrophic fungus Ustilago maydis. Mol. Microbiol. 89:179-188.

Roberts, A., and Pachter, L. 2013. Streaming fragment assignment for realtime analysis of sequencing experiments. Nat. Methods 10:71-73.

Roberts, A., Trapnell, C., Donaghey, J., Rinn, J. L., and Pachter, L. 2011. Improving RNA-seq expression estimates by correcting for fragment bias. Genome Biol. 12:R22.

Rubery, P. H., and Fosket, D. E. 1969. Changes in phenylalanine ammonialyase activity during xylem differentiation in Coleus and soybean. Planta 87:54-62.
Sanders, I. R. 2011. Mycorrhizal symbioses: How to be seen as a good fungus. Curr. Biol. 21:R550-R552.

Singh, K., Foley, R. C., and Oñate-Sánchez, L. 2002. Transcription factors in plant defense and stress responses. Curr. Opin. Plant Biol. 5: 430-436.

Song, Y., You, J., and Xiong, L. 2009. Characterization of OsIAA1 gene, a member of rice Aux/IAA family involved in auxin and brassinosteroid hormone responses and plant morphogenesis. Plant Mol. Biol. 70: 297-309.

Thrower, L. B., and Chan, Y. 1980. Gau sun: A cultivated host-parasite combination from China. Econ. Bot. 34:20-26.

Ton, J., Flors, V., and Mauch-Mani, B. 2009. The multifaceted role of ABA in disease resistance. Trends Plant Sci. 14:310-317.

Trapnell, C., Williams, B. A., Pertea, G., Mortazavi, A., Kwan, G., van Baren, M. J., Salzberg, S. L., Wold, B. J., and Pachter, L. 2010 Transcript assembly and quantification by RNA-seq reveals unannotated transcripts and isoform switching during cell differentiation. Nat. Biotechnol. 28:511-515.

Tuteja, N. 2007. Abscisic acid and abiotic stress signaling. Plant Signal. Behav. 2:135-138

Waikhom, S. D., Louis, B., Roy, P., Singh, W. M., Bharwaj, P. K., and Talukdar, N. C. 2013. Scanning electron microscopy of pollen structure throws light on resolving Bambusa-Dendrocalamus complex: Bamboo flowering evidence. Plant Syst. Evol. 300:1261-1268.

Wang, X., Guo, C., Peng, J., Li, C., Wan, F., Zhang, S., Zhou, Y., Yan, Y., Qi, L., Sun, K., Yang, S., Gong, Z., and Li, J. 2019. ABRE-BINDING FACTORS play a role in the feedback regulation of ABA signaling by mediating rapid ABA induction of ABA co-receptor genes. New Phytol. 221:341-355.

Wang, Z. D., Yan, N., Wang, Z. H., Zhang, X. H., Zhang, J. Z., Xue, H. M., Wang, L. X., Zhan, Q., Xu, Y. P., and Guo, D. P. 2017. RNA-seq analysis provides insight into reprogramming of culm development in Zizania latifolia induced by Ustilago esculenta. Plant Mol. Biol. 95:533-547.

Wilkinson, S., Kudoyarova, G. R., Veselov, D. S., Arkhipova, T. N., and Davies, W. J. 2012. Plant hormone interactions: Innovative targets for crop breeding and management. J. Exp. Bot. 63:3499-3509.

Yan, N., Du, Y., Liu, X., Chu, C., Shi, J., Zhang, H., Liu, Y., and Zhang, Z. 2018. Morphological characteristics, nutrients, and bioactive compounds of Zizania latifolia, and health benefits of its seeds. Molecules 23:1561.

Yan, N., Wang, X. Q., Xu, X. F., Guo, D. P., Wang, Z. D., Zhang, J. Z., Hyde, K. D., and Liu, H. L. 2013. Plant growth and photosynthetic performance of Zizania latifolia are altered by endophytic Ustilago esculenta infection. Physiol. Mol. Plant Pathol. 83:75-83.

Yang, C., Zhang, X., Li, J., Bao, M., Ni, D., and Seago, J. L. 2014. Anatomy and histochemistry of roots and shoots in wild rice (Zizania latifolia Griseb.). J. Bot. 2014:1-9.

You, W., Liu, Q., Zou, K., Yu, X., Cui, H., and Ye, Z. 2011. Morphological and molecular differences in two strains of Ustilago esculenta. Curr. Microbiol. 62:44-54

Young, M. D., Wakefield, M. J., Smyth, G. K., and Oshlack, A. 2010. Gene ontology analysis for RNA-seq: Accounting for selection bias. Genome Biol. 11:R14.

Yuan, J., Chen, D., Ren, Y., Zhang, X., and Zhao, J. 2008. Characteristic and expression analysis of a metallothionein gene, $O s M T 2 b$, down-regulated by cytokinin suggests functions in root development and seed embryo germination of rice. Plant Physiol. 146:1637-1650.

Zafari, S., Niknam, V., Musetti, R., and Noorbakhsh, S. N. 2012. Effect of phytoplasma infection on metabolite content and antioxidant enzyme activity in lime (Citrus aurantifolia). Acta Physiol. Plant. 34:561-568.

Zhang, J. Z., Chu, F. Q., Guo, D. P., Hyde, K. D., and Xie, G. L. 2012. Cytology and ultrastructure of interactions between Ustilago esculenta and Zizania latifolia. Mycol. Prog. 11:499-508.

Zhang, J. Z., Chu, F. Q., Guo, D. P., Ojaghian, M. R., and Hyde, K. D. 2014 The vacuoles containing multivesicular bodies: A new observation in interaction between Ustilago esculenta and Zizania latifolia. Eur. J. Plant Pathol. 138:79-91. 